Supporting Information

\title{
Aerobic Oxidative C-H Azolation of Indoles and One-Pot Synthesis of Azolyl Thioindoles by Flavin-Iodine-Coupled Organocatalysis
}

Kazumasa Tanimoto, Hayaki Okai, Marina Oka, Ryoma Ohkado, and Hiroki Iida*

Department of Chemistry, Graduate School of Natural Science and Technology,

Shimane University, 1060 Nishikawatsu, Matsue, 690-8504, Japan

E-mail address: iida@riko.shimane-u.ac.jp

$\begin{array}{ll}\text { 1. General } & \text { S2 }\end{array}$

2. Materials $\quad \mathrm{S} 2$

3. Optimization of Reaction Condition and Experimental Procedure S3

4. ${ }^{1} \mathrm{H}$ and ${ }^{13} \mathrm{C}\left\{{ }^{1} \mathrm{H}\right\}$ NMR Spectra of Novel Compounds $\quad$ S11

5. ${ }^{1} \mathrm{H}$ and ${ }^{13} \mathrm{C}\left\{{ }^{1} \mathrm{H}\right\}$ NMR Spectra of Known Compounds $\quad$ S23

$\begin{array}{lr}\text { 6. References } & \text { S30 }\end{array}$ 


\section{General}

Melting points (M.p.) were determined on a SANSYO SMP-300 (SANSYO, Tokyo, Japan) and are uncorrected. The IR spectra were recorded on a JASCO FT/IR-660plus spectrophotometer (JASCO, Tokyo, Japan). The NMR spectra were measured using JEOL JNM-L400 and JNM ECX500 spectrometers (JEOL, Akishima, Japan) operating at 400 and $500 \mathrm{MHz}$, respectively, for ${ }^{1} \mathrm{H}$ and 100 and $126 \mathrm{MHz}$, respectively, for ${ }^{13} \mathrm{C}$ using tetramethylsilane (TMS) or a solvent residual peak as the internal standard. The electrospray ionization mass (ESI-MS) spectra were recorded using a Bruker microTOFII-SHIY3 mass spectrometer (Bruker, Billerica, MA). The GC measurements were performed on a Shimadzu GC-2014 gas chromatograph (Shimadzu, Kyoto, Japan) equipped with a flame ionization detector (FID) using Supelco Equity-5 (30 m x 0.25 mm) column.

\section{Materials}

All starting materials were purchased from Aldrich (Milwaukee, WI), Wako Pure Chemical Industries (Osaka, Japan), and Tokyo Kasei (TCI, Tokyo, Japan) and were used as received. Riboflavin tetraacetate (13b), ${ }^{\mathrm{S} 1}$ 5-ethyl-1,3,7,8-tetramethylalloxazinium triflate $(\mathbf{6} \cdot \mathbf{T f O}),{ }^{\mathrm{S} 2} 1,10$ ethylene-7,8-dimethylisoalloxazinium chloride $(\mathbf{1 4} \cdot \mathbf{C l}),{ }^{\mathrm{S} 2}$ and 5-ethyl-10-(2-hydroxylethyl)-7,8dimethylisoalloxazinium triflate $(\mathbf{1 5} \cdot \mathbf{T f O})^{\mathrm{S} 2}$ were synthesized according to the previously reported methods. 


\section{Optimization of Reaction Condition and Experimental Procedure}

\section{Effects of Solvents and Iodine Sources}

The reaction condition for the aerobic oxidative cross-coupling of 1a with 2a was optimized as shown in Table S1.

Table S1. Screening of solvents and iodine sources ${ }^{a}$

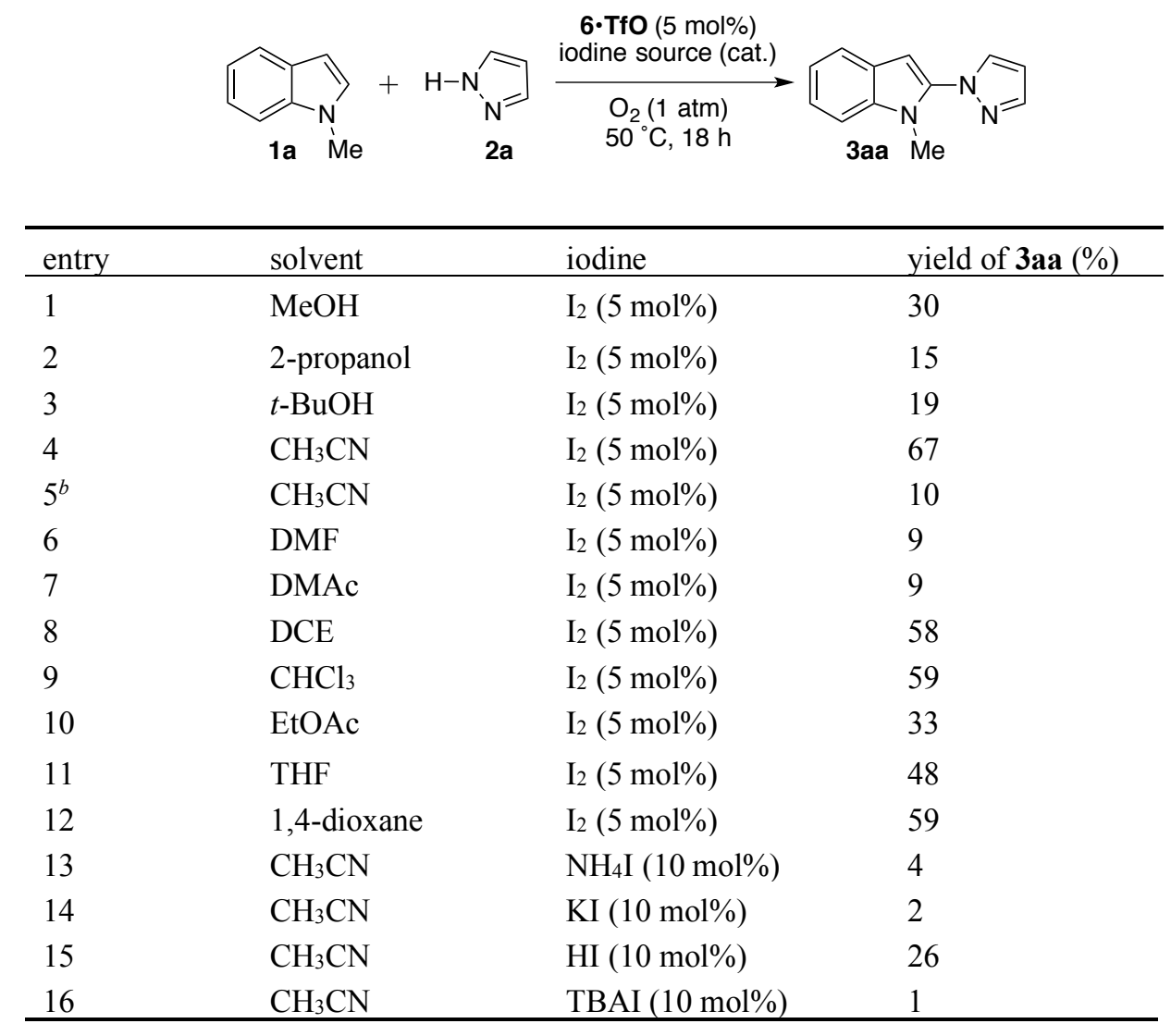

${ }^{a}$ Conditions: 1a $(0.5 \mathrm{M}), \mathbf{2 a}(1.0 \mathrm{M})$, 6•TfO $(5 \mathrm{~mol} \%)$, iodine source $(5$ or $10 \mathrm{~mol} \%)$, and solvent under $\mathrm{O}_{2}\left(1 \mathrm{~atm}\right.$, balloon) at $50{ }^{\circ} \mathrm{C}$ for $18 \mathrm{~h}$. Yield was determined by ${ }^{1} \mathrm{H}$ NMR or GC measurement. ${ }^{b} 25^{\circ} \mathrm{C}$ 


\section{Effects of Catalysts}

To examine the catalytic activity of diverse riboflavin-derived catalysts for the aerobic oxidative cross-coupling of 1a with $\mathbf{2 a}$, we undertook a study to compare the reactivities of related catalysts (Table S2). Among the seven flavins, 6•TfO was the most efficient catalyst.

Table S2. Comparison with catalytic activity of flavins for the reaction of $\mathbf{1 a}$ with $\mathbf{2} \mathbf{a}^{a}$
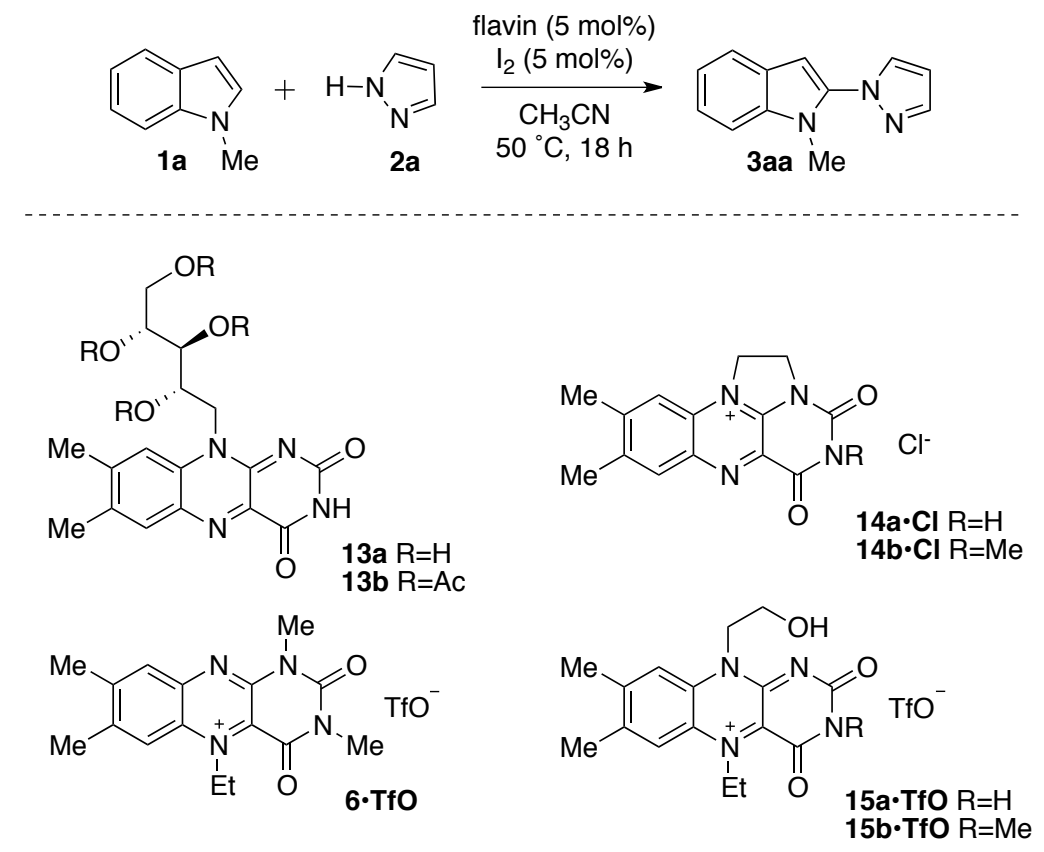

\begin{tabular}{ccc}
\hline entry & flavin & yield (\%) \\
\hline 1 & $\mathbf{1 3 a ^ { b }}$ & 9 \\
2 & $\mathbf{1 3 b}$ & 10 \\
3 & $\mathbf{1 4 a} \cdot \mathbf{C l}$ & 19 \\
4 & $\mathbf{1 4 b} \cdot \mathbf{C l}$ & 25 \\
5 & $\mathbf{6 \bullet T f O}$ & 67 \\
4 & $\mathbf{1 5 a} \cdot \mathbf{T f O}$ & 20 \\
5 & $\mathbf{1 5 b} \cdot \mathbf{T f O}$ & 29 \\
\hline
\end{tabular}

${ }^{a}$ Conditions: 1a $(0.5 \mathrm{M}), \mathbf{2 a}(1.0 \mathrm{M})$, flavin $(5 \mathrm{~mol} \%), \mathrm{I}_{2}(5 \mathrm{~mol} \%)$, and $\mathrm{CH}_{3} \mathrm{CN}$ under $\mathrm{O}_{2}\left(1 \mathrm{~atm}\right.$, balloon) at $50{ }^{\circ} \mathrm{C}$ for $18 \mathrm{~h}$. Yield was determined by ${ }^{1} \mathrm{H}$ NMR or GC measurement. ${ }^{b} \mathbf{1 3 a}$ was insoluble in $\mathrm{CH}_{3} \mathrm{CN}$. 
Typical Procedure for Catalytic Cross-Coupling of 1a with 2a. A mixture of 1a (125 $\mu \mathrm{L}, 1.0$ mmol), 2a (136 mg, $2.0 \mathrm{mmol}), \mathrm{I}_{2}(12.7 \mathrm{mg}, 0.050 \mathrm{mmol})$, and $\mathbf{6} \cdot \mathbf{T f O}(22.5 \mathrm{mg}, 0.050 \mathrm{mmol})$ in $\mathrm{CH}_{3} \mathrm{CN}(1.0 \mathrm{~mL})$ was stirred under $\mathrm{O}_{2}(1 \mathrm{~atm}$, balloon $)$ at $50{ }^{\circ} \mathrm{C}$ for $36 \mathrm{~h}$. After the solvent was removed by evaporation, the residue was purified by column chromatography $\left(\mathrm{SiO}_{2}\right.$, chloroform) to give 3aa (143 mg, 73\%) as a white solid. The results are summarized in Scheme 2.

Spectroscopic data of $3 a a .{ }^{1} \mathrm{H}$ NMR $\left(500 \mathrm{MHz}, \mathrm{CDCl}_{3}, 25{ }^{\circ} \mathrm{C}, \delta\right): 7.80(\mathrm{~d}, J=1.2 \mathrm{~Hz}, 1 \mathrm{H}), 7.70$ $(\mathrm{d}, J=2.3 \mathrm{~Hz}, 1 \mathrm{H}), 7.62(\mathrm{~d}, J=8.0 \mathrm{~Hz}, 1 \mathrm{H}), 7.34(\mathrm{~d}, J=8.2 \mathrm{~Hz}, 1 \mathrm{H}), 7.28(\mathrm{t}, J=7.6 \mathrm{~Hz}, 1 \mathrm{H}), 7.16$ (t, $J=7.3 \mathrm{~Hz}, 1 \mathrm{H}), 6.50(\mathrm{~s}, 1 \mathrm{H}), 6.45(\mathrm{t}, J=2.1 \mathrm{~Hz}, 1 \mathrm{H}), 3.66(\mathrm{~s}, 3 \mathrm{H}) \cdot{ }^{13} \mathrm{C}\left\{{ }^{1} \mathrm{H}\right\} \mathrm{NMR}(126 \mathrm{MHz}$, $\left.\mathrm{CDCl}_{3}, 25{ }^{\circ} \mathrm{C}\right): \delta 141.9,135.8,132.5,126.2,122.7,121.1,120.5,109.7,106.9,95.9,30.0$. The spectral property of 3aa was in good agreement with the reported data. ${ }^{\mathrm{S} 3}$

Spectroscopic data of $3 \mathbf{b a}$. Column chromatography $\left(\mathrm{SiO}_{2}\right.$, hexane/dichloromethane $=2 / 1$ to $1 / 1$, $\mathrm{v} / \mathrm{v}$ ) afforded the desired product $\left(116 \mathrm{mg}, 80 \%\right.$ ) as a pale yellow oil. IR (neat, $\mathrm{cm}^{-1}$ ): 2958, 2931, 1586, 1567, 1472, 1457, 749, 447. ${ }^{1} \mathrm{H}$ NMR (500 MHz, $\left.\mathrm{CDCl}_{3}, 25^{\circ} \mathrm{C}, \delta\right): 7.79$ (dd, $J=1.9,0.6 \mathrm{~Hz}$, $1 \mathrm{H}), 7.70(\mathrm{dd}, J=2.3,0.7 \mathrm{~Hz}, 1 \mathrm{H}), 7.62(\mathrm{~d}, J=7.8 \mathrm{~Hz}, 1 \mathrm{H}), 7.37(\mathrm{dd}, J=8.4,0.7 \mathrm{~Hz}, 1 \mathrm{H}), 7.29-$ $7.26(\mathrm{~m}, 1 \mathrm{H}), 7.17-7.14(\mathrm{~m}, 1 \mathrm{H}),, 6.50(\mathrm{~d}, J=0.6 \mathrm{~Hz}, 1 \mathrm{H}), 6.45(\mathrm{t}, J=2.3 \mathrm{~Hz}, 1 \mathrm{H}), 4.13(\mathrm{t}, J=7.5$ $\mathrm{Hz}, 2 \mathrm{H}), 1.66-1.60(\mathrm{~m}, 2 \mathrm{H}), 1.18(\mathrm{qt}, J=7.5,7.4 \mathrm{~Hz}, 2 \mathrm{H}), 0.80(\mathrm{t}, J=7.4 \mathrm{~Hz}, 3 \mathrm{H}) .{ }^{13} \mathrm{C}\left\{{ }^{1} \mathrm{H}\right\} \mathrm{NMR}$ (126 MHz, $\left.\mathrm{CDCl}_{3}, 25^{\circ} \mathrm{C}, \delta\right): 141.8,135.4,135.1,132.6,126.2,122.5,121.2,120.3,110.1,106.8$, 96.3, 43.3, 31.9, 20.1, 13.7. HRMS (ESI+) $(\mathrm{m} / \mathrm{z}):\left(\mathrm{M}+\mathrm{H}^{+}\right)$calculated for $\mathrm{C}_{15} \mathrm{H}_{18} \mathrm{~N}_{3}, 240.1495$; found, 240.1484 .

Spectroscopic data of 3ca. Column chromatography $\left(\mathrm{SiO}_{2}\right.$, hexane/ethyl acetate $=4 / 1$ to $\left.1 / 1, \mathrm{v} / \mathrm{v}\right)$ afforded the desired product (101 mg, 75\%) as a yellow oil. IR (neat, $\mathrm{cm}^{-1}$ ): 2937, 1568, 1446, 1362, 1033, 785, 747. ${ }^{1} \mathrm{H}$ NMR (500 MHz, $\mathrm{CDCl}_{3}, 25^{\circ} \mathrm{C}, \delta$ ): $7.76(\mathrm{dd}, J=9.3,2.1 \mathrm{~Hz}, 2 \mathrm{H}), 7.42$ $(\mathrm{d}, J=7.9 \mathrm{~Hz}, 1 \mathrm{H}), 7.06(\mathrm{t}, J=7.5 \mathrm{~Hz}, 1 \mathrm{H}), 6.96(\mathrm{dd}, J=7.2,0.8 \mathrm{~Hz}, 1 \mathrm{H}), 6.44(\mathrm{t}, J=1.1 \mathrm{~Hz}, 1 \mathrm{H})$, $6.40(\mathrm{~s}, 1 \mathrm{H}), 4.21(\mathrm{t}, J=5.8 \mathrm{~Hz}, 2 \mathrm{H}), 2.98(\mathrm{t}, J=6.1 \mathrm{~Hz}, 2 \mathrm{H}), 2.21(\mathrm{q}, J=6.0,5.9 \mathrm{~Hz}, 2 \mathrm{H}) .{ }^{13} \mathrm{C}\left\{{ }^{1} \mathrm{H}\right\}$ $\operatorname{NMR}\left(126 \mathrm{MHz}, \mathrm{CDCl}_{3}, 25^{\circ} \mathrm{C}, \delta\right): 141.7,135.0,132.9,131.5,124.3,122.2,120.5,119.4,118.1$, 106.9, 93.4, 43.1, 24.9, 22.9. HRMS (ESI+) $(\mathrm{m} / \mathrm{z}):\left(\mathrm{M}+\mathrm{H}^{+}\right)$calculated for $\mathrm{C}_{14} \mathrm{H}_{14} \mathrm{~N}_{3}, 224.1182$; found, 224.1178 .

Spectroscopic data of $3 d \boldsymbol{d a}$. Column chromatography $\left(\mathrm{SiO}_{2}\right.$, hexane/dichloromethane $=1 / 1$ to $1 / 2$, $\mathrm{v} / \mathrm{v})$ afforded the desired product $(106 \mathrm{mg}, 58 \%)$ as a white solid. Mp: 100.8-101.9 ${ }^{\circ} \mathrm{C}$. IR $(\mathrm{KBr}$, $\left.\mathrm{cm}^{-1}\right): 3119,2952,2836,1485,1475,1452,1224,1165,764 .{ }^{1} \mathrm{H}$ NMR $\left(500 \mathrm{MHz}, \mathrm{CDCl}_{3}, 25^{\circ} \mathrm{C}\right.$, 
$\delta): 7.77(\mathrm{~d}, J=1.9 \mathrm{~Hz}, 1 \mathrm{H}), 7.58(\mathrm{~d}, J=2.2 \mathrm{~Hz}, 1 \mathrm{H}), 7.22-7.16(\mathrm{~m}, 4 \mathrm{H}), 7.10(\mathrm{~d}, J=2.4 \mathrm{~Hz}, 1 \mathrm{H})$, $6.96(\mathrm{~d}, J=6.3 \mathrm{~Hz}, 2 \mathrm{H}), 6.88(\mathrm{dd}, J=8.9,2.4 \mathrm{~Hz}, 1 \mathrm{H}), 6.51(\mathrm{~s}, 1 \mathrm{H}), 6.39(\mathrm{t}, J=2.2 \mathrm{~Hz}, 1 \mathrm{H}), 5.32$ (s, 2H), $3.85(\mathrm{~s}, 3 \mathrm{H}) .{ }^{13} \mathrm{C}\left\{{ }^{1} \mathrm{H}\right\} \mathrm{NMR}\left(126 \mathrm{MHz}, \mathrm{CDCl}_{3}, 25^{\circ} \mathrm{C}, \delta\right): 154.8,141.9,137.5,136.0,132.4$, 130.6, 128.6, 127.4 126.8, 126.6, 113.2, 111.4, 106.9, 102.8, 96.5, 55.8, 47.1. HRMS $(\mathrm{ESI}+)(\mathrm{m} / \mathrm{z})$ : $\left(\mathrm{M}+\mathrm{H}^{+}\right)$calculated for $\mathrm{C}_{19} \mathrm{H}_{18} \mathrm{~N}_{3} \mathrm{O}, 304.1444$; found, 304.1451 .

Spectroscopic data of 3ea. Column chromatography $\left(\mathrm{SiO}_{2}\right.$, chloroform/methanol $=100 / 0$ to $99 / 1$, $\mathrm{v} / \mathrm{v})$ afforded the desired product $(92.2 \mathrm{mg}, 77 \%)$ as a pale orange solid. ${ }^{1} \mathrm{H} \mathrm{NMR}\left(500 \mathrm{MHz}, \mathrm{CDCl}_{3}\right.$, $\left.25^{\circ} \mathrm{C}, \delta\right): 9.49(\mathrm{~s}, 1 \mathrm{H}), 7.90(\mathrm{~d}, J=2.6 \mathrm{~Hz}, 1 \mathrm{H}), 7.74(\mathrm{~d}, J=1.8 \mathrm{~Hz}, 1 \mathrm{H}), 7.55(\mathrm{~d}, J=7.6 \mathrm{~Hz}, 1 \mathrm{H})$, 7.28-7.26 (m, 1H), 7.19-7.12 (m, 2H), 6.50-6.49 (m, 1H), $2.40(\mathrm{~s}, 3 \mathrm{H}) .{ }^{13} \mathrm{C}\left\{{ }^{1} \mathrm{H}\right\}$ NMR (126 MHz, $\left.\mathrm{CDCl}_{3}, 25{ }^{\circ} \mathrm{C}, \delta\right): 140.7,133.1,131.6,129.5,128.7,122.5,120.0,118.9,111.0,107.5,98.3,8.8$. The spectral property of 3 ea was in good agreement with the reported data. ${ }^{\mathrm{S} 3}$

Spectroscopic data of $3 \mathbf{f a}$. Column chromatography ( $\mathrm{SiO}_{2}$, chloroform) afforded the desired product $(88.3 \mathrm{mg}, 69 \%)$ as a white solid. ${ }^{1} \mathrm{H}$ NMR $\left(500 \mathrm{MHz}\right.$, DMSO- $\left.d_{6}, 25^{\circ} \mathrm{C}, \delta\right): 11.68(\mathrm{~s}, 1 \mathrm{H})$, 8.40 (d, $J=2.3 \mathrm{~Hz}, 1 \mathrm{H}), 7.78$ (d, $J=1.5 \mathrm{~Hz}, 1 \mathrm{H}), 7.27$ (d, $J=8.7 \mathrm{~Hz}, 1 \mathrm{H}), 7.02$ (d, $J=2.4 \mathrm{~Hz}$, $1 \mathrm{H}), 6.73(\mathrm{dd}, J=8.7,2.5 \mathrm{~Hz}, 1 \mathrm{H}), 6.56(\mathrm{t}, J=2.1 \mathrm{~Hz}, 1 \mathrm{H}), 6.51(\mathrm{~d}, J=1.5 \mathrm{~Hz}, 1 \mathrm{H}), 3.75(\mathrm{~s}, 3 \mathrm{H})$. ${ }^{13} \mathrm{C}\left\{{ }^{1} \mathrm{H}\right\}$ NMR (126 MHz, DMSO- $\left.d_{6}, 25{ }^{\circ} \mathrm{C}, \delta\right): 153.9,140.8,136.2,128.7,128.4,127.9,112.1$, $110.9,107.7,101.9,87.5,55.3$. The spectral property of $\mathbf{3 f a}$ was in good agreement with the reported data. ${ }^{\mathrm{S} 4}$

Spectroscopic data of $\mathbf{3 g}$. Column chromatography $\left(\mathrm{SiO}_{2}\right.$, chloroform/methanol $=100 / 0$ to $99 / 1$, $\mathrm{v} / \mathrm{v}$ ) afforded the desired product (105 mg, $73 \%$ ) as a pale yellow solid. Mp: $91.6-93.3^{\circ} \mathrm{C}$. IR ( $\mathrm{KBr}$, $\left.\mathrm{cm}^{-1}\right): 2999,2953,2929,1585,1490,1449,1211,1140,836,816 .{ }^{1} \mathrm{H}$ NMR $\left(500 \mathrm{MHz}, \mathrm{CDCl}_{3}\right.$, $\left.25^{\circ} \mathrm{C}, \delta\right): 7.59(\mathrm{~s}, 1 \mathrm{H}), 7.45(\mathrm{~s}, 1 \mathrm{H}), 7.20(\mathrm{~d}, J=8.8 \mathrm{~Hz}, 1 \mathrm{H}), 7.05(\mathrm{~d}, J=2.4 \mathrm{~Hz}, 1 \mathrm{H}), 6.92(\mathrm{dd}, J$ $=8.9,2.5 \mathrm{~Hz}, 1 \mathrm{H}), 6.36(\mathrm{~s}, 1 \mathrm{H}), 3.83(\mathrm{~s}, 3 \mathrm{H}), 3.63(\mathrm{~s}, 3 \mathrm{H}), 2.15(\mathrm{~s}, 3 \mathrm{H}) .{ }^{13} \mathrm{C}\left\{{ }^{1} \mathrm{H}\right\} \mathrm{NMR}(126 \mathrm{MHz}$, $\left.\mathrm{CDCl}_{3}, 25^{\circ} \mathrm{C}, \delta\right): 154.6,142.6,136.4,131.0,130.7,126.5,117.3,112.7,110.5,102.6,95.1,55.9$, 30.1, 8.8. HRMS $(\mathrm{ESI}+)(\mathrm{m} / \mathrm{z})$ : $\left(\mathrm{M}+\mathrm{H}^{+}\right)$calculated for $\mathrm{C}_{14} \mathrm{H}_{16} \mathrm{~N}_{3} \mathrm{O}, 242.1288$; found, 242.1287 .

Spectroscopic data of $3 \boldsymbol{h b}$. Column chromatography ( $\mathrm{SiO}_{2}$, chloroform) afforded the desired product (113 mg, $65 \%$ ) as a white solid. Mp: $131.4-132.5^{\circ} \mathrm{C}$. IR $\left(\mathrm{KBr}, \mathrm{cm}^{-1}\right): 2949,2925,1587$, 1553, 1474, 1332, 957, 798, 789, 601. ${ }^{1} \mathrm{H}$ NMR $\left(500 \mathrm{MHz}, \mathrm{CDCl}_{3}, 25^{\circ} \mathrm{C}, \delta\right): 7.72(\mathrm{~d}, J=1.8 \mathrm{~Hz}$, 1H), $7.62(\mathrm{~s}, 1 \mathrm{H}), 7.50$ (s, 1H), 7.35 (dd, $J=8.6,1.9 \mathrm{~Hz}, 1 \mathrm{H}), 7.21$ (d, $J=8.7 \mathrm{~Hz}, 1 \mathrm{H}), 6.39$ (s, 1H), $3.69(\mathrm{~s}, 3 \mathrm{H}), 2.18(\mathrm{~s}, 3 \mathrm{H}) .{ }^{13} \mathrm{C}\left\{{ }^{1} \mathrm{H}\right\} \mathrm{NMR}\left(126 \mathrm{MHz}, \mathrm{CDCl}_{3}, 25{ }^{\circ} \mathrm{C}, \delta\right): 143.1,137.0,134.5$, $130.8,127.9,125.4,123.4,117.8,113.6,111.3,94.8,30.4,8.9$. HRMS $(\mathrm{ESI}+)(\mathrm{m} / \mathrm{z}):\left(\mathrm{M}+\mathrm{H}^{+}\right)$ 
calculated for $\mathrm{C}_{13} \mathrm{H}_{13} \mathrm{BrN}_{3}, 290.0287$; found, 290.0286.

Spectroscopic data of $3 \mathbf{a b}$. Column chromatography ( $\mathrm{SiO}_{2}$, chloroform) afforded the desired product $(90.3 \mathrm{mg}, 71 \%)$ as a white solid. Mp: $95.8-97.3{ }^{\circ} \mathrm{C}$. IR $\left(\mathrm{KBr}, \mathrm{cm}^{-1}\right): 2925,1585,1559$, 1480, 1455, 1012, 959, 749, 612. ${ }^{1} \mathrm{H}$ NMR $\left(500 \mathrm{MHz}, \mathrm{CDCl}_{3}, 25^{\circ} \mathrm{C}, \delta\right): 7.60(\mathrm{t}, J=3.9 \mathrm{~Hz}, 2 \mathrm{H})$, $7.46(\mathrm{~s}, 1 \mathrm{H}), 7.32(\mathrm{~d}, J=8.0 \mathrm{~Hz}, 1 \mathrm{H}), 7.26(\mathrm{td}, J=7.6,1.1 \mathrm{~Hz}, 1 \mathrm{H}), 7.15(\mathrm{td}, J=7.5,1.0 \mathrm{~Hz}, 1 \mathrm{H})$, $6.44(\mathrm{~s}, 1 \mathrm{H}), 3.66(\mathrm{~s}, 3 \mathrm{H}), 2.15(\mathrm{~s}, 3 \mathrm{H}) .{ }^{13} \mathrm{C}\left\{{ }^{1} \mathrm{H}\right\} \mathrm{NMR}\left(126 \mathrm{MHz}, \mathrm{CDCl}_{3}, 25^{\circ} \mathrm{C}, \delta\right): 142.7,136.1$, 135.8, 130.8, 126.2, 122.5, 121.0, 120.4, 117.4, 109.7, 95.4, 30.0, 8.8. HRMS (ESI+) $(m / z):(\mathrm{M}+$ $\mathrm{H}^{+}$) calculated for $\mathrm{C}_{13} \mathrm{H}_{14} \mathrm{~N}_{3}, 212.1182$; found, 212.1186.

Spectroscopic data of 3ac. Column chromatography $\left(\mathrm{SiO}_{2}\right.$, chloroform/methanol $=100 / 0$ to $99 / 1$, $\mathrm{v} / \mathrm{v})$ afforded the desired product (79.8 $\mathrm{mg}, 67 \%)$ as a pale yellow solid. ${ }^{1} \mathrm{H} \mathrm{NMR}\left(500 \mathrm{MHz}, \mathrm{CDCl}_{3}\right.$, $\left.25{ }^{\circ} \mathrm{C}, \delta\right): 8.38(\mathrm{~s}, 1 \mathrm{H}), 8.20(\mathrm{~s}, 1 \mathrm{H}), 7.65(\mathrm{~d}, J=7.9 \mathrm{~Hz}, 1 \mathrm{H}), 7.38-7.32(\mathrm{~m}, 2 \mathrm{H}), 7.22-7.19(\mathrm{~m}$, $1 \mathrm{H}), 6.60(\mathrm{~s}, 1 \mathrm{H}), 3.65(\mathrm{~s}, 3 \mathrm{H}) .{ }^{13} \mathrm{C}\left\{{ }^{1} \mathrm{H}\right\} \mathrm{NMR}\left(126 \mathrm{MHz}, \mathrm{CDCl}_{3}, 25^{\circ} \mathrm{C}, \delta\right): 153.3,145.7,136.1$, $131.3,125.8,123.5,121.5,120.9,109.9,97.6,30.0$. The spectral property of 3ac was in good agreement with the reported data. ${ }^{\mathrm{S} 4}$

Spectroscopic data of $3 c$. Column chromatography $\left(\mathrm{SiO}_{2}\right.$, hexane/ethyl acetate $=2 / 1$ to $\left.1 / 1, \mathrm{v} / \mathrm{v}\right)$ afforded the desired product (106 mg, 79\%) as a pale yellow solid. Mp: $101.3-103.2{ }^{\circ} \mathrm{C}$. IR $(\mathrm{KBr}$, $\left.\mathrm{cm}^{-1}\right): 2955,2930,1563,1487,1456,1328,1000,793,747,675 .{ }^{1} \mathrm{H}$ NMR $\left(500 \mathrm{MHz}, \mathrm{CDCl}_{3}\right.$, $\left.25^{\circ} \mathrm{C}, \delta\right): 8.40(\mathrm{~s}, 1 \mathrm{H}), 8.16(\mathrm{~s}, 1 \mathrm{H}), 7.44(\mathrm{~d}, J=7.9 \mathrm{~Hz}, 1 \mathrm{H}), 7.09(\mathrm{t}, J=7.5 \mathrm{~Hz}, 1 \mathrm{H}), 7.01(\mathrm{~d}, J=$ $7.1 \mathrm{~Hz}, 1 \mathrm{H}$ ), $6.50(\mathrm{~s}, 1 \mathrm{H}), 4.13(\mathrm{t}, J=5.7 \mathrm{~Hz}, 2 \mathrm{H}), 2.99(\mathrm{t}, J=6.1 \mathrm{~Hz}, 2 \mathrm{H}), 2.22$ (quin, $J=6.0 \mathrm{~Hz}$, 2H). ${ }^{13} \mathrm{C}\left\{{ }^{1} \mathrm{H}\right\}$ NMR $\left(126 \mathrm{MHz}, \mathrm{CDCl}_{3}, 25^{\circ} \mathrm{C}, \delta\right): 153.0,144.8,133.2,130.4,123.9,122.3,120.9$, 120.2, 118.5, 95.3, 42.7, 24.6, 22.7. HRMS $(\mathrm{ESI}+)(\mathrm{m} / \mathrm{z}):\left(\mathrm{M}+\mathrm{H}^{+}\right)$calculated for $\mathrm{C}_{13} \mathrm{H}_{13} \mathrm{~N}_{4}$, 225.1135; found, 225.1133 .

Spectroscopic data of 3ad. Column chromatography ( $\mathrm{SiO}_{2}$, chloroform) afforded the desired product $(131 \mathrm{mg}, 88 \%)$ as a white solid. ${ }^{1} \mathrm{H}$ NMR $\left(500 \mathrm{MHz}, \mathrm{CDCl}_{3} 25{ }^{\circ} \mathrm{C}, \delta\right): 8.04(\mathrm{~s}, 1 \mathrm{H}), 7.91$ $(\mathrm{d}, J=7.6 \mathrm{~Hz}, 1 \mathrm{H}), 7.70(\mathrm{~d}, J=8.0 \mathrm{~Hz}, 1 \mathrm{H}), 7.43-7.31(\mathrm{~m}, 4 \mathrm{H}), 7.31-7.20(\mathrm{~m}, 2 \mathrm{H}), 6.66(\mathrm{~s}, 1 \mathrm{H})$, 3.49 (s, 3H). ${ }^{13} \mathrm{C}\left\{{ }^{1} \mathrm{H}\right\}$ NMR $\left(126 \mathrm{MHz}, \mathrm{CDCl}_{3}, 25{ }^{\circ} \mathrm{C}, \delta\right): 143.9,143.5,136.0,135.8,130.5,126.4$, $124.5,123.32,123.29,121.4,120.9,120.8,110.7,109.9,99.3$ 29.5. The spectral property of 3ad was in good agreement with the reported data. ${ }^{\mathrm{S} 3}$

Spectroscopic data of 7aa. Column chromatography $\left(\mathrm{SiO}_{2}\right.$, hexane/ethyl acetate $\left.=4 / 1, \mathrm{v} / \mathrm{v}\right)$ afforded the desired product $(17.7 \mathrm{mg}, 9 \%)$ as a white solid. ${ }^{1} \mathrm{H}$ NMR $\left(500 \mathrm{MHz}, \mathrm{CDCl}_{3}, 25{ }^{\circ} \mathrm{C}\right.$, $\delta): 7.87(\mathrm{~d}, J=1.7 \mathrm{~Hz}, 1 \mathrm{H}), 7.80(\mathrm{~d}, J=2.5 \mathrm{~Hz}, 1 \mathrm{H}), 7.49$ (d, $J=7.9 \mathrm{~Hz}, 1 \mathrm{H}), 7.39-7.34(\mathrm{~m}, 1 \mathrm{H})$, 
$7.32(\mathrm{~d}, J=8.2 \mathrm{~Hz}, 1 \mathrm{H}), 7.29-7.24(\mathrm{~m}, 1 \mathrm{H}), 6.54(\mathrm{t}, J=2.1 \mathrm{~Hz}, 1 \mathrm{H}), 3.63(\mathrm{~s}, 3 \mathrm{H}) .{ }^{13} \mathrm{C}\left\{{ }^{1} \mathrm{H}\right\} \mathrm{NMR}$ $\left(126 \mathrm{MHz}, \mathrm{CDCl}_{3}, 25^{\circ} \mathrm{C}, \delta\right): 142.4,136.4,135.8,133.5,128.7,124.2,122.1,121.4,110.2,107.1$, $54.5,30.6$. The spectral property of $7 \mathbf{a a}$ was in good agreement with the reported data. ${ }^{\mathrm{S}}$

Typical Procedure for Catalytic One-Pot Three-Component Synthesis of 5aaa. A mixture of 1a (74.8 $\mu \mathrm{L}, 0.60 \mathrm{mmol}), \mathbf{2 a}(81.8 \mathrm{mg}, 1.2 \mathrm{mmol}), \mathrm{I}_{2}(7.60 \mathrm{mg}, 0.030 \mathrm{mmol})$, and 6•TfO (13.4 mg, $0.030 \mathrm{mmol})$ in $\mathrm{CH}_{3} \mathrm{CN}(0.6 \mathrm{~mL})$ was stirred under $\mathrm{O}_{2}\left(1 \mathrm{~atm}\right.$, balloon) at $50{ }^{\circ} \mathrm{C}$ for $36 \mathrm{~h}$. To the reaction mixture, $4 \mathbf{a}(90.3 \mathrm{mg}, 0.727 \mathrm{mmol})$ was added and the mixture was stirred at $50{ }^{\circ} \mathrm{C}$ for 24 h. After the solvent was removed by evaporation, the residue was purified by column chromatography $\left(\mathrm{SiO}_{2}\right.$, hexane/ethyl acetate $\left.=4 / 1, \mathrm{v} / \mathrm{v}\right)$ to give $\mathbf{5 a a a}(144 \mathrm{mg}, 75 \%)$ as a white solid. The results are summarized in Scheme 3.

Spectroscopic data of 5aaa. Mp: 148.9-150.1 ${ }^{\circ} \mathrm{C}$. IR (KBr, cm $\left.{ }^{-1}\right)$ : 2923, 1561, 1491, 1477, 1391, 1037, 940, 802, 748. ${ }^{1} \mathrm{H}$ NMR $\left(500 \mathrm{MHz}, \mathrm{CDCl}_{3}, 25^{\circ} \mathrm{C}, \delta\right): 7.81(\mathrm{~d}, J=1.8 \mathrm{~Hz}, 1 \mathrm{H}), 7.70-7.64(\mathrm{~m}$, $2 \mathrm{H}), 7.39(\mathrm{~d}, J=8.3 \mathrm{~Hz}, 1 \mathrm{H}), 7.34(\mathrm{td}, J=7.6,1.1 \mathrm{~Hz}, 1 \mathrm{H}), 7.20(\mathrm{td}, J=7.5,1.0 \mathrm{~Hz}, 1 \mathrm{H}), 7.00-$ $6.91(\mathrm{~m}, 4 \mathrm{H}), 6.41(\mathrm{t}, J=2.2 \mathrm{~Hz}, 1 \mathrm{H}), 3.72(\mathrm{~s}, 3 \mathrm{H}), 2.22(\mathrm{~s}, 3 \mathrm{H}) .{ }^{13} \mathrm{C}\left\{{ }^{1} \mathrm{H}\right\} \mathrm{NMR}\left(126 \mathrm{MHz}, \mathrm{CDCl}_{3}\right.$, $\left.25^{\circ} \mathrm{C}, \delta\right): 142.3,139.0,135.3,135.0,134.9,133.6,129.8,128.2,126.5,123.8,121.6,120.3,110.1$, 106.8, 96.2, 30.7, 21.0. HRMS (ESI+) $(\mathrm{m} / \mathrm{z})$ : $\left(\mathrm{M}+\mathrm{H}^{+}\right)$calculated for $\mathrm{C}_{19} \mathrm{H}_{18} \mathrm{~N}_{3} \mathrm{~S}, 320.1216$; found, 320.1221 .

Spectroscopic data of 5aab. Column chromatography $\left(\mathrm{SiO}_{2}\right.$, hexane/ethyl acetate $=6 / 1$, v/v) afforded the desired product (123 mg, $67 \%$ ) as a white solid. Mp: $108.5-110.1{ }^{\circ} \mathrm{C}$. IR $\left(\mathrm{KBr}, \mathrm{cm}^{-1}\right)$ : $1558,1478,1448,1394,1038,940,739 .{ }^{1} \mathrm{H}$ NMR $\left(500 \mathrm{MHz}, \mathrm{CDCl}_{3}, 25{ }^{\circ} \mathrm{C}, \delta\right): 7.83$ (d, $J=1.5$ $\mathrm{Hz}, 1 \mathrm{H}), 7.69-7.65(\mathrm{~m}, 2 \mathrm{H}), 7.43(\mathrm{~d}, J=8.0 \mathrm{~Hz}, 1 \mathrm{H}), 7.37$ (td, $J=7.0,1.0 \mathrm{~Hz}, 1 \mathrm{H}), 7.23$ (t, $J=7.5$ $\mathrm{Hz}, 1 \mathrm{H}), 7.17-7.12(\mathrm{~m}, 2 \mathrm{H}), 7.08-7.03(\mathrm{~m}, 3 \mathrm{H}), 6.44(\mathrm{t}, J=2.0 \mathrm{~Hz}, 1 \mathrm{H}), 3.76(\mathrm{~s}, 3 \mathrm{H}) .{ }^{13} \mathrm{C}\left\{{ }^{1} \mathrm{H}\right\}$ NMR $\left(126 \mathrm{MHz}, \mathrm{CDCl}_{3}, 25^{\circ} \mathrm{C}, \delta\right): 142.4,139.1,138.6,135.4,133.6,129.0,128.2,126.1,125.2$, 123.9, 121.7, 120.3, 110.2, 106.9, 95.7, 30.8. HRMS $(\mathrm{ESI}+)(\mathrm{m} / \mathrm{z}):\left(\mathrm{M}+\mathrm{H}^{+}\right)$calculated for $\mathrm{C}_{18} \mathrm{H}_{16} \mathrm{~N}_{3} \mathrm{~S}, 306.1059$; found, 306.1061 .

Spectroscopic data of 5aac. Column chromatography $\left(\mathrm{SiO}_{2}\right.$, hexane/ethyl acetate $=8 / 1$, v/v) afforded the desired product (129 mg, 63\%) as a colorless oil. IR (neat, $\mathrm{cm}^{-1}$ ): 2952, 2925, 2853, 1560, 1477, 1454, 941, 743. ${ }^{1} \mathrm{H}$ NMR $\left(500 \mathrm{MHz}, \mathrm{CDCl}_{3}, 25^{\circ} \mathrm{C}, \delta\right): 7.87-7.84(\mathrm{~m}, 2 \mathrm{H}), 7.81(\mathrm{~d}, J$ $=7.5 \mathrm{~Hz}, 1 \mathrm{H}), 7.38-7.32(\mathrm{~m}, 2 \mathrm{H}), 7.28-7.24(\mathrm{~m}, 1 \mathrm{H}), 6.52(\mathrm{t}, J=2.3 \mathrm{~Hz}, 1 \mathrm{H}), 3.65(\mathrm{~s}, 3 \mathrm{H}), 2.51(\mathrm{t}$, $J=7.3 \mathrm{~Hz}, 2 \mathrm{H}), 1.35-1.10(\mathrm{~m}, 12 \mathrm{H}), 0.86(\mathrm{t}, J=7.3 \mathrm{~Hz}, 3 \mathrm{H}) .{ }^{13} \mathrm{C}\left\{{ }^{1} \mathrm{H}\right\} \mathrm{NMR}\left(126 \mathrm{MHz}, \mathrm{CDCl}_{3}\right.$, 
$\left.25^{\circ} \mathrm{C}, \delta\right): 142.1,138.3,135.1,133.9,128.5,123.6,121.1,120.2,110.0,106.6,99.7,36.6,31.9$, 30.4, 29.7, 29.3, 29.2, 28.5, 22.8, 14.2. HRMS (ESI+) $(\mathrm{m} / \mathrm{z}):\left(\mathrm{M}+\mathrm{H}^{+}\right)$calculated for $\mathrm{C}_{20} \mathrm{H}_{28} \mathrm{~N}_{3} \mathrm{~S}$, 342.1998; found, 342.2006.

Spectroscopic data of $\mathbf{5 c c a}$. Column chromatography $\left(\mathrm{SiO}_{2}\right.$, dichloromethane) afforded the desired product (160 mg, $78 \%$ ) as a green solid. Mp: 100.2-102.3 ${ }^{\circ} \mathrm{C}$. IR (KBr, cm $\left.{ }^{-1}\right): 3106,2941,1547$, 1491, 1440, 1377, 805. ${ }^{1} \mathrm{H}$ NMR (500 MHz, $\left.\mathrm{CDCl}_{3}, 25^{\circ} \mathrm{C}, \delta\right): 8.42(\mathrm{~s}, 1 \mathrm{H}), 8.17$ (s, 1H), 7.51 (d, $J=8.0 \mathrm{~Hz}, 1 \mathrm{H}), 7.16(\mathrm{t}, J=7.5 \mathrm{~Hz}, 1 \mathrm{H}), 7.09$ (d, $J=7.0 \mathrm{~Hz}, 1 \mathrm{H}), 6.98(\mathrm{~s}, 4 \mathrm{H}), 4.23(\mathrm{t}, J=5.5 \mathrm{~Hz}$, 2H), $3.06(\mathrm{t}, J=6.0 \mathrm{~Hz}, 2 \mathrm{H}), 2.29$ (quin, $J=5.0 \mathrm{~Hz}, 2 \mathrm{H}$ ), $2.25(\mathrm{~s}, 3 \mathrm{H}) .{ }^{13} \mathrm{C}\left\{{ }^{1} \mathrm{H}\right\} \mathrm{NMR}(126 \mathrm{MHz}$, $\mathrm{CDCl}_{3}, 25^{\circ} \mathrm{C}, \delta$ ): $153.0,146.1,135.4,134.5,133.6,132.5,129.9,126.6,126.5,122.7,121.9,121.2$, 117.7, 96.1, 43.4, 24.5, 22.6, 20.9. HRMS (ESI+) $(\mathrm{m} / \mathrm{z})$ : $\left(\mathrm{M}+\mathrm{H}^{+}\right)$calculated for $\mathrm{C}_{20} \mathrm{H}_{19} \mathrm{~N}_{4} \mathrm{~S}$, 347.1325; found, 347.1332 .

Spectroscopic data of $\mathbf{5 c c c}$. Column chromatography $\left(\mathrm{SiO}_{2}\right.$, dichloromethane) afforded the desired product (141 mg, 64\%) as a green oil. IR (neat, $\mathrm{cm}^{-1}$ ): 2952, 2925, 2854, 1551, 1454, 1442, 998, 748. ${ }^{1} \mathrm{H}$ NMR $\left(500 \mathrm{MHz}, \mathrm{CDCl}_{3}, 25^{\circ} \mathrm{C}, \delta\right): 8.64(\mathrm{~s}, 1 \mathrm{H}), 8.20(\mathrm{~s}, 1 \mathrm{H}), 7.60(\mathrm{~d}, J=8.0 \mathrm{~Hz}, 1 \mathrm{H})$, $7.17(\mathrm{t}, J=7.3 \mathrm{~Hz}, 1 \mathrm{H}), 7.06$ (dd, $J=7.1,0.9 \mathrm{~Hz}, 1 \mathrm{H}), 4.13(\mathrm{t}, J=5.7 \mathrm{~Hz}, 2 \mathrm{H}), 3.02(\mathrm{t}, J=6.1 \mathrm{~Hz}$, $2 \mathrm{H}$ ), 2.55 (t, $J=7.2 \mathrm{~Hz}, 2 \mathrm{H}$ ), 2.24 (quin, $J=6.0 \mathrm{~Hz}, 2 \mathrm{H}$ ), 1.36-1.15 (m, 12H), 0.86 (t, $J=7.2 \mathrm{~Hz}$, $3 \mathrm{H}) .{ }^{13} \mathrm{C}\left\{{ }^{1} \mathrm{H}\right\}$ NMR $\left(126 \mathrm{MHz}, \mathrm{CDCl}_{3}, 25^{\circ} \mathrm{C}, \delta\right): 152.9,146.4,133.1,132.4,126.6,122.6,121.5$, 121.0, 117.7, 99.3, 43.0, 36.7, 31.9, 29.8, 29.23, 29.16, 28.4, 24.6, 22.7, 22.6, 14.2. HRMS (ESI+) $(\mathrm{m} / \mathrm{z}):\left(\mathrm{M}+\mathrm{H}^{+}\right)$calculated for $\mathrm{C}_{21} \mathrm{H}_{29} \mathrm{~N}_{4} \mathrm{~S}, 369.2107$; found, 369.2106 .

Typical Procedure for Catalytic One-Pot Three-Component Synthesis of IIIb. A mixture of 1i (35.2 mg, $0.30 \mathrm{mmol}), \mathbf{2 c}(82.8 \mathrm{mg}, 1.2 \mathrm{mmol}), \mathrm{I}_{2}(3.81 \mathrm{mg}, 0.015 \mathrm{mmol})$, and 6•TfO (6.70 mg, $0.015 \mathrm{mmol})$ in 1,4 -dioxane $(0.3 \mathrm{~mL})$ was stirred under $\mathrm{O}_{2}(1 \mathrm{~atm}$, balloon $)$ at $80{ }^{\circ} \mathrm{C}$ for $72 \mathrm{~h}$. To the reaction mixture, $4 \mathbf{d}(72.0 \mathrm{mg}, 0.36 \mathrm{mmol})$ was added and the mixture was stirred at $80{ }^{\circ} \mathrm{C}$ for $24 \mathrm{~h}$. After the solvent was removed by evaporation, the residue was purified by column chromatography $\left(\mathrm{SiO}_{2}\right.$, hexane/ethyl acetate $\left.=1 / 1, \mathrm{v} / \mathrm{v}\right)$ to give $\mathbf{I I I b}(89.6 \mathrm{mg}, 78 \%)$ as a pale yellow solid.

Spectroscopic data of IIIb. ${ }^{1} \mathrm{H}$ NMR $\left(500 \mathrm{MHz}\right.$, DMSO-d $\left.6,25^{\circ} \mathrm{C}, \delta\right): 12.75(\mathrm{~s}, 1 \mathrm{H}), 9.13(\mathrm{~s}, 1 \mathrm{H})$, $8.41(\mathrm{~s}, 1 \mathrm{H}), 7.58(\mathrm{~d}, J=7.9 \mathrm{~Hz}, 1 \mathrm{H}), 7.53(\mathrm{~d}, J=8.2 \mathrm{~Hz}, 1 \mathrm{H}), 7.30(\mathrm{t}, J=7.2 \mathrm{~Hz}, 1 \mathrm{H}), 7.20(\mathrm{t}, J$ $=7.4 \mathrm{~Hz}, 1 \mathrm{H}), 6.37(\mathrm{~s}, 2 \mathrm{H}), 3.57(\mathrm{~s}, 6 \mathrm{H}), 3.57(\mathrm{~s}, 3 \mathrm{H}) .{ }^{13} \mathrm{C}\left\{{ }^{1} \mathrm{H}\right\} \mathrm{NMR}\left(126 \mathrm{MHz}\right.$, DMSO- $d_{6}, 25{ }^{\circ} \mathrm{C}$, $\delta): 153.3,152.6,145.2,135.9,134.8,133.6,131.7,128.3,123.7,121.3,119.0,112.5,103.9,92.8$, 
$60.0,55.8$. The spectral property of IIIb was in good agreement with the reported data. ${ }^{\mathrm{S}}$ 


\section{4. ${ }^{1} \mathrm{H}$ and ${ }^{13} \mathrm{C}\left\{{ }^{1} \mathrm{H}\right\}$ NMR Spectra of Novel Compounds}<smiles>CC(C)(C)n1c(-n2cccn2)cc2ccccc21</smiles>
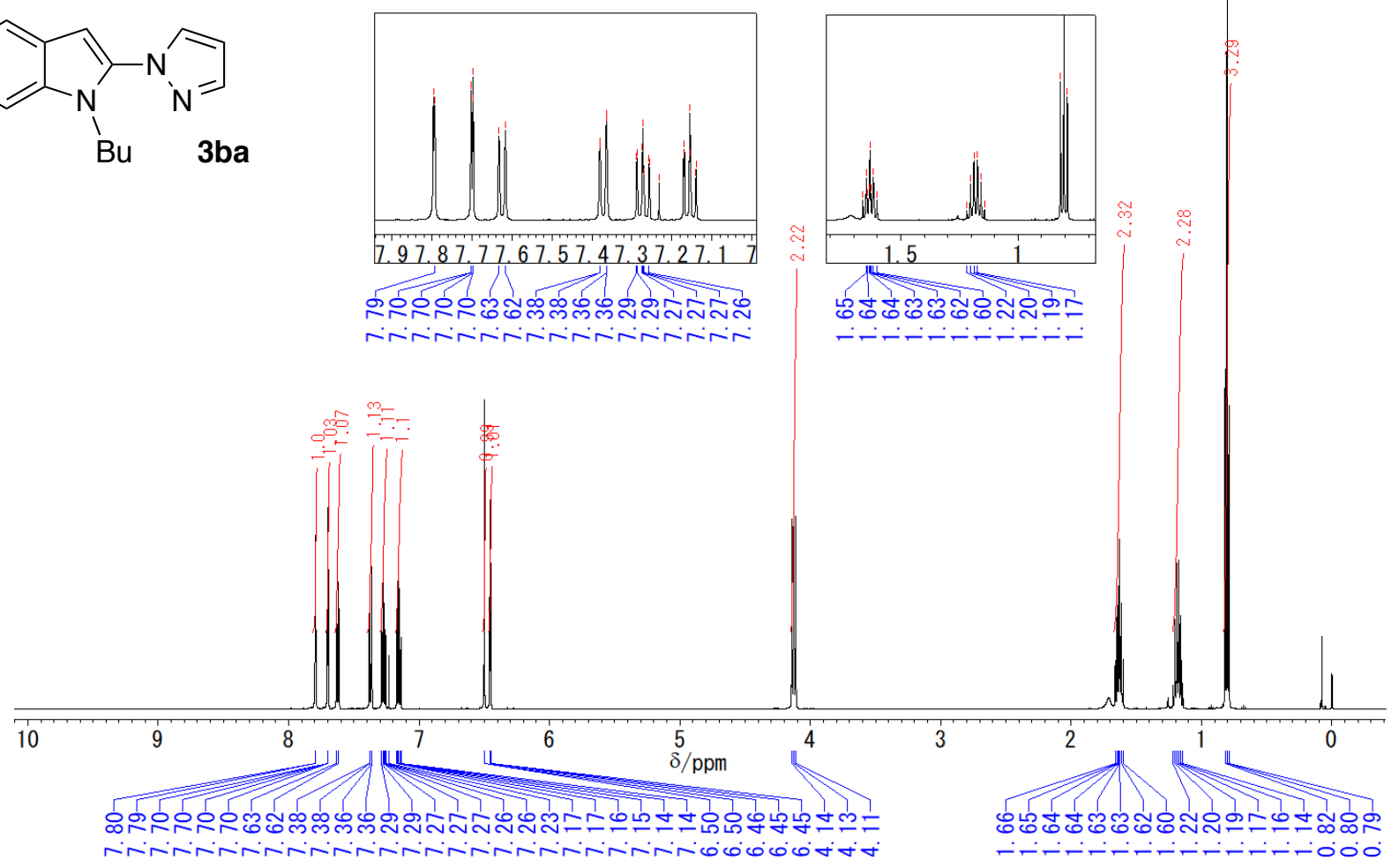

Spectrum S1. ${ }^{1} \mathrm{H}$ NMR $\left(\mathrm{CDCl}_{3}, 500 \mathrm{MHz}\right)$ spectrum of compound $\mathbf{3 b a}$.

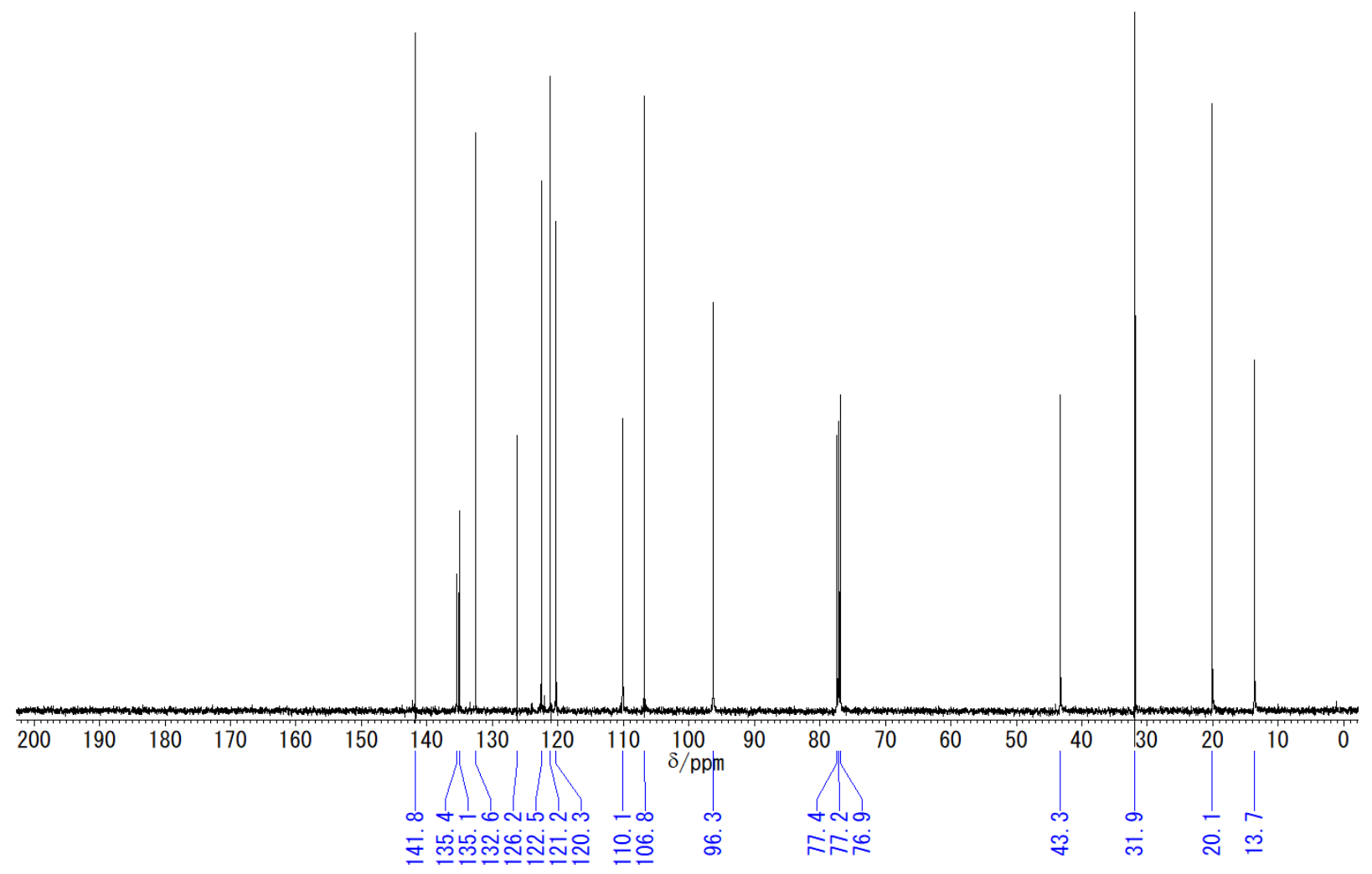

Spectrum S2. ${ }^{13} \mathrm{C}\left\{{ }^{1} \mathrm{H}\right\} \mathrm{NMR}\left(\mathrm{CDCl}_{3}, 126 \mathrm{MHz}\right)$ spectrum of compound $3 \mathbf{b a}$. 


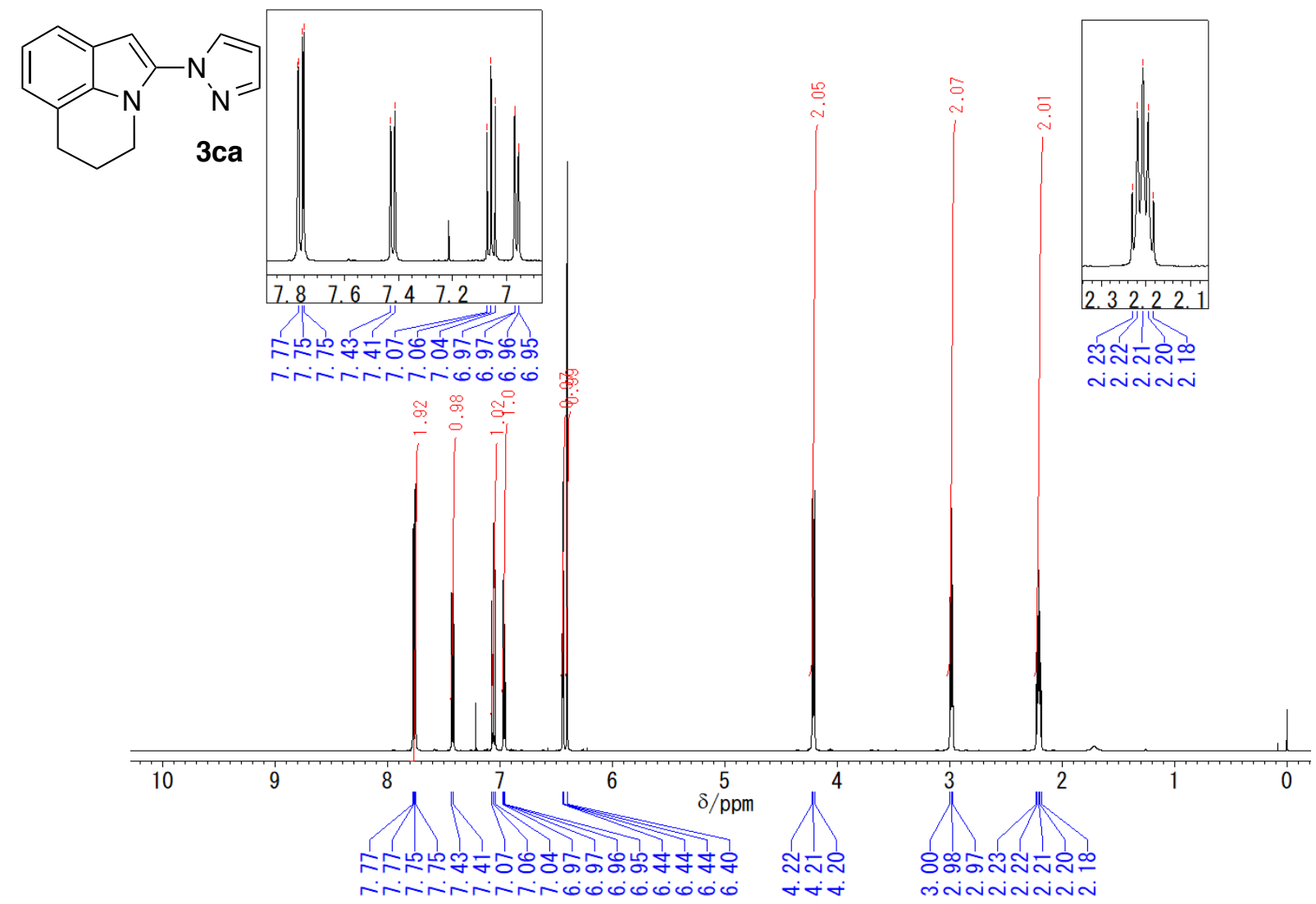

Spectrum S3. ${ }^{1} \mathrm{H}$ NMR $\left(\mathrm{CDCl}_{3}, 500 \mathrm{MHz}\right)$ spectrum of compound $3 \mathbf{c a}$.

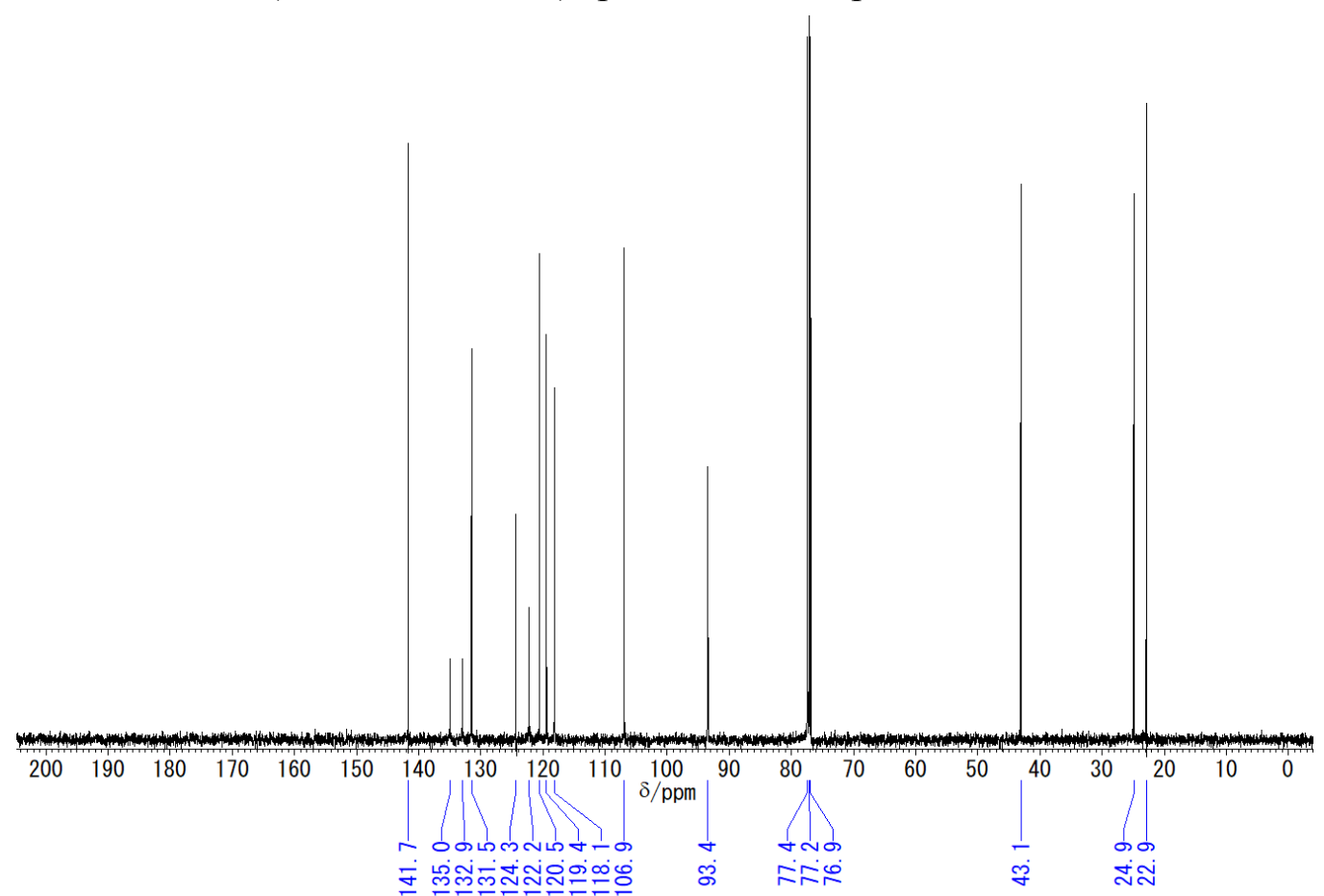

Spectrum S4. ${ }^{13} \mathrm{C}\left\{{ }^{1} \mathrm{H}\right\}$ NMR $\left(\mathrm{CDCl}_{3}, 126 \mathrm{MHz}\right)$ spectrum of compound 3ca. 


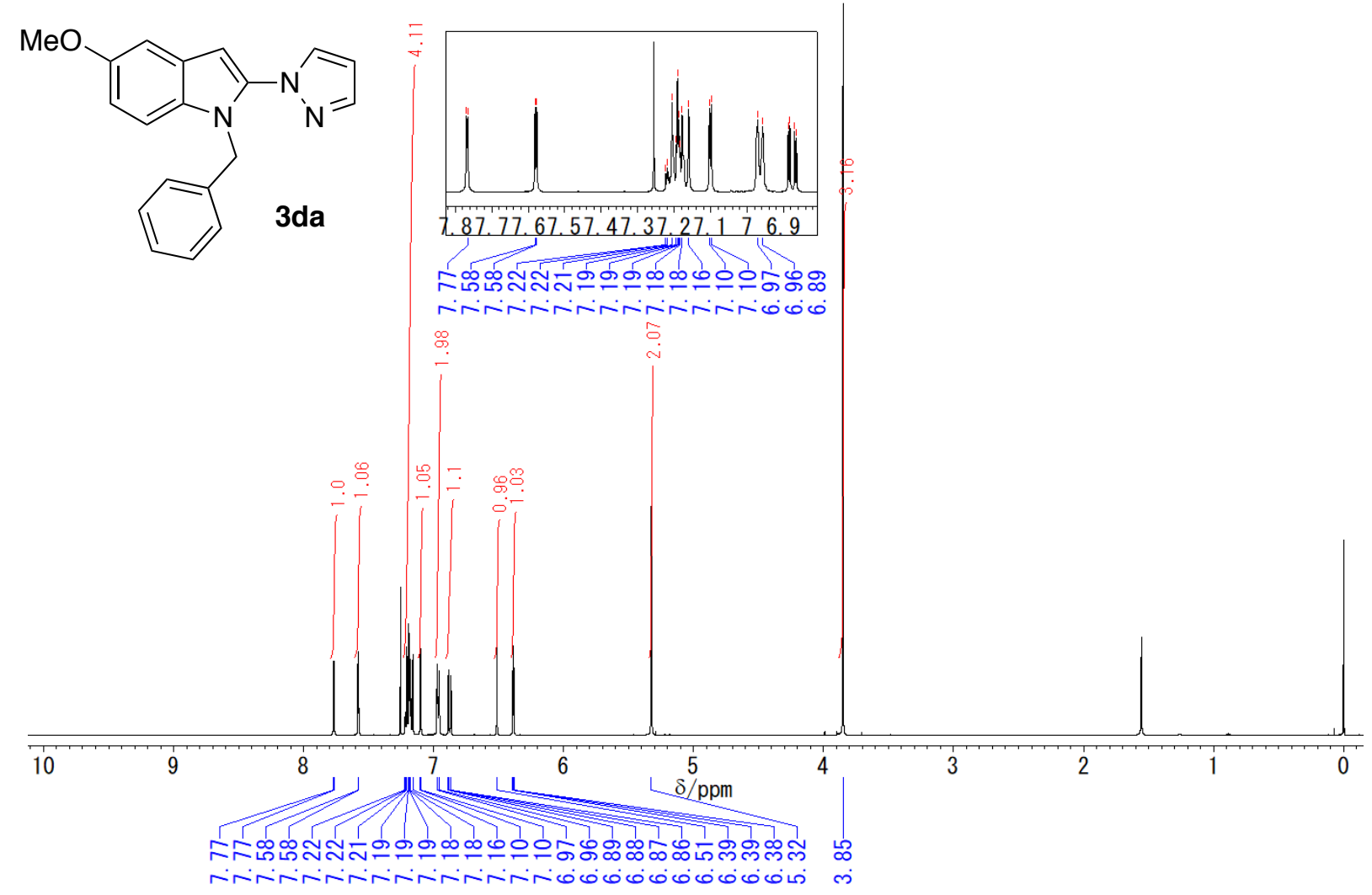

Spectrum S5. ${ }^{1} \mathrm{H}$ NMR $\left(\mathrm{CDCl}_{3}, 500 \mathrm{MHz}\right)$ spectrum of compound 3da.

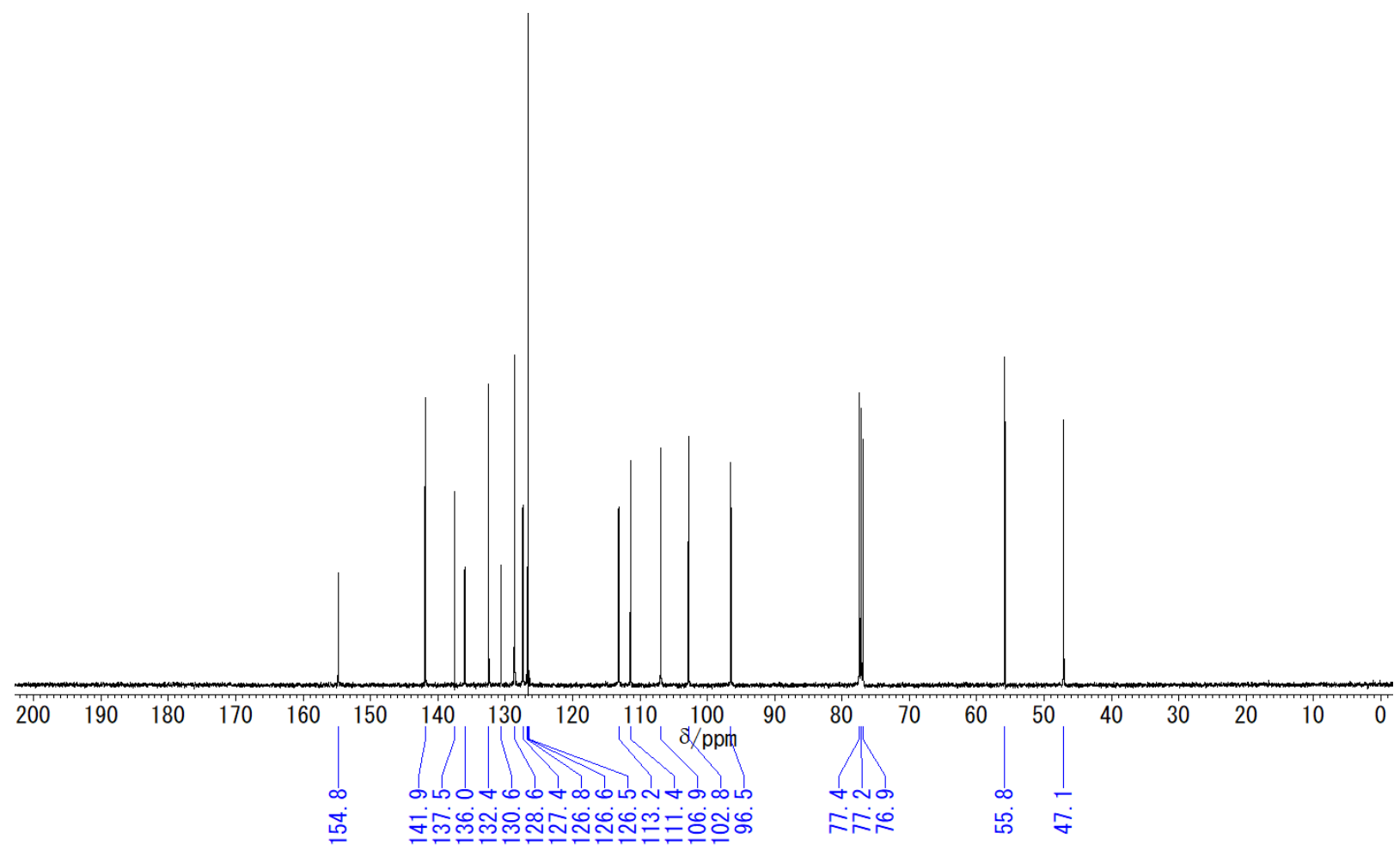

Spectrum S6. ${ }^{13} \mathrm{C}\left\{{ }^{1} \mathrm{H}\right\} \mathrm{NMR}\left(\mathrm{CDCl}_{3}, 126 \mathrm{MHz}\right)$ spectrum of compound 3da. 
<smiles>COc1ccc2c(c1)cc(-n1cc(C)cn1)n2C</smiles>
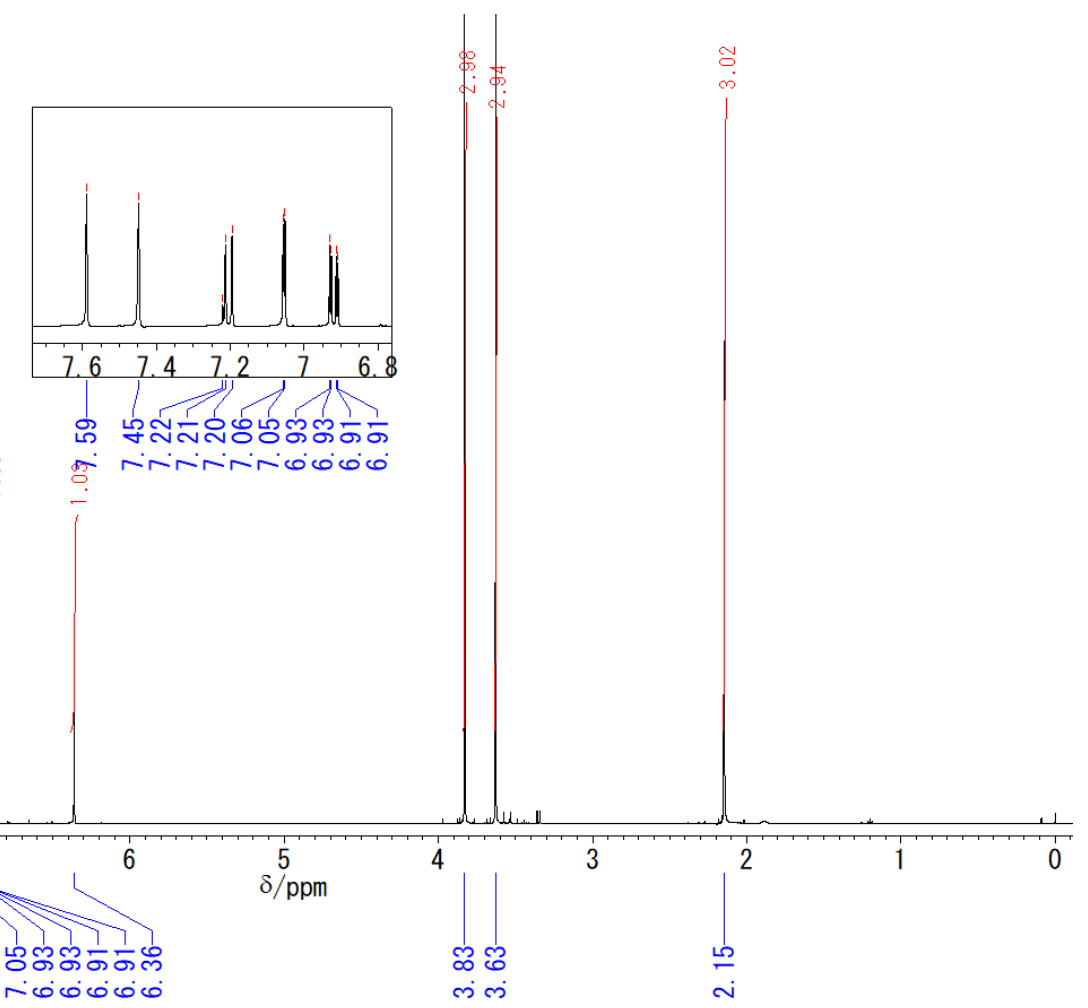

Spectrum S7. ${ }^{1} \mathrm{H}$ NMR $\left(\mathrm{CDCl}_{3}, 500 \mathrm{MHz}\right)$ spectrum of compound $\mathbf{3 g b}$.

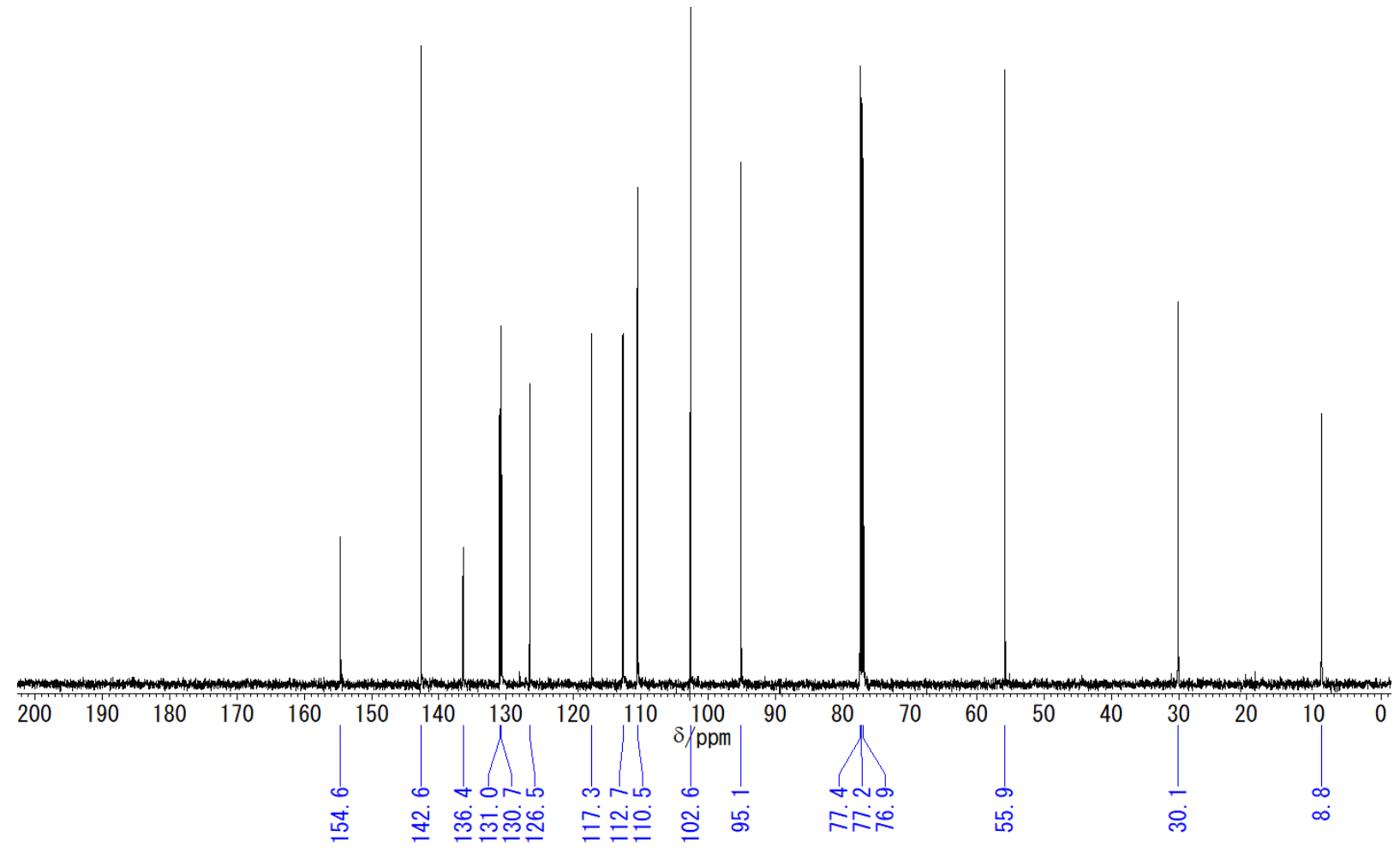

Spectrum S8. ${ }^{13} \mathrm{C}\left\{{ }^{1} \mathrm{H}\right\}$ NMR $\left(\mathrm{CDCl}_{3}, 126 \mathrm{MHz}\right)$ spectrum of compound $\mathbf{3 g b}$. 


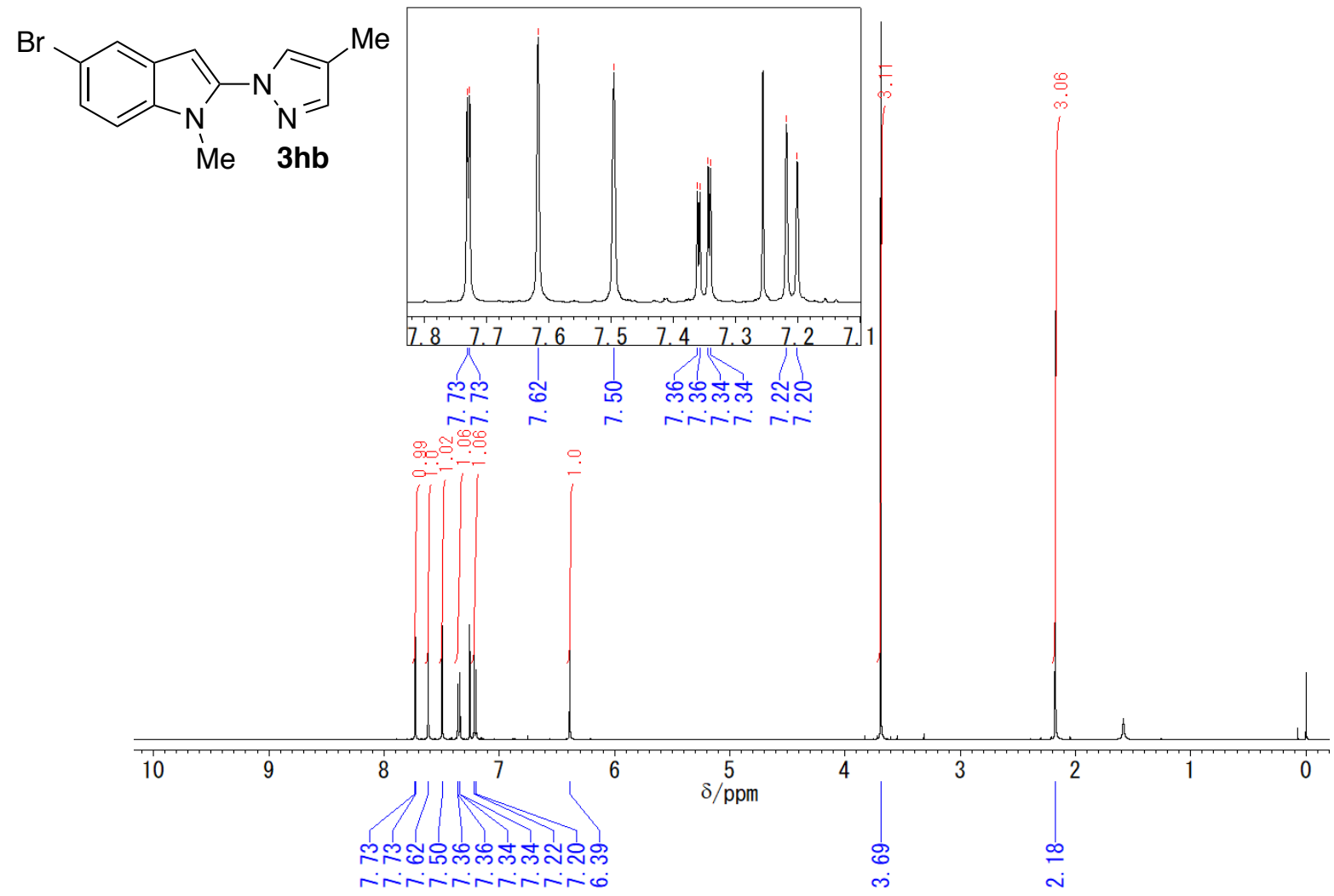

Spectrum S9. ${ }^{1} \mathrm{H} \mathrm{NMR}\left(\mathrm{CDCl}_{3}, 500 \mathrm{MHz}\right)$ spectrum of compound $\mathbf{3 h b}$.

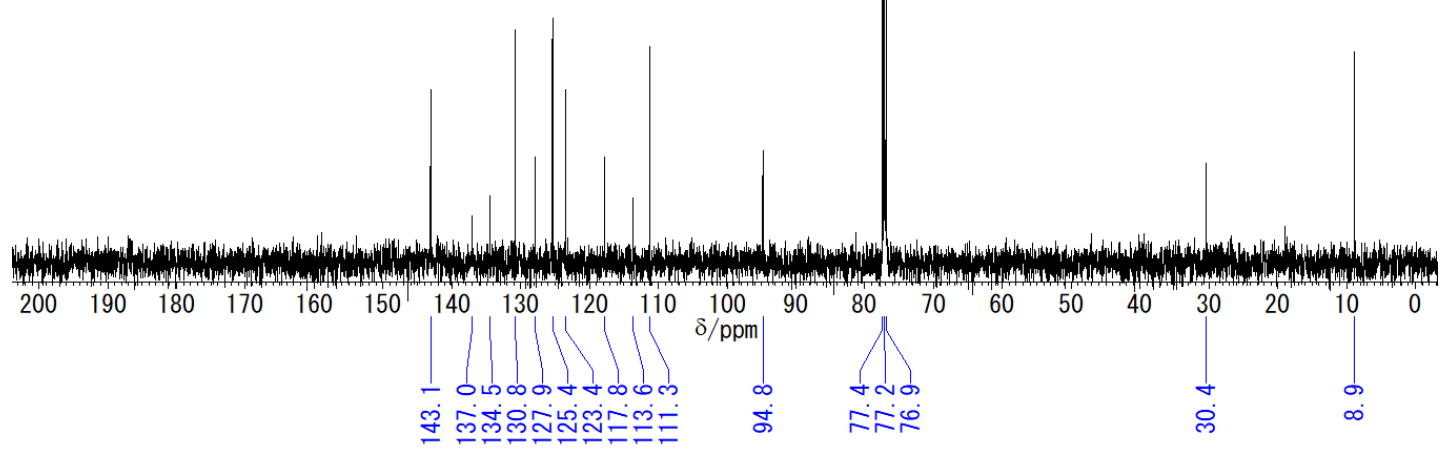

Spectrum S10. ${ }^{13} \mathrm{C}\left\{{ }^{1} \mathrm{H}\right\} \mathrm{NMR}\left(\mathrm{CDCl}_{3}, 126 \mathrm{MHz}\right)$ spectrum of compound $\mathbf{3 h b}$. 


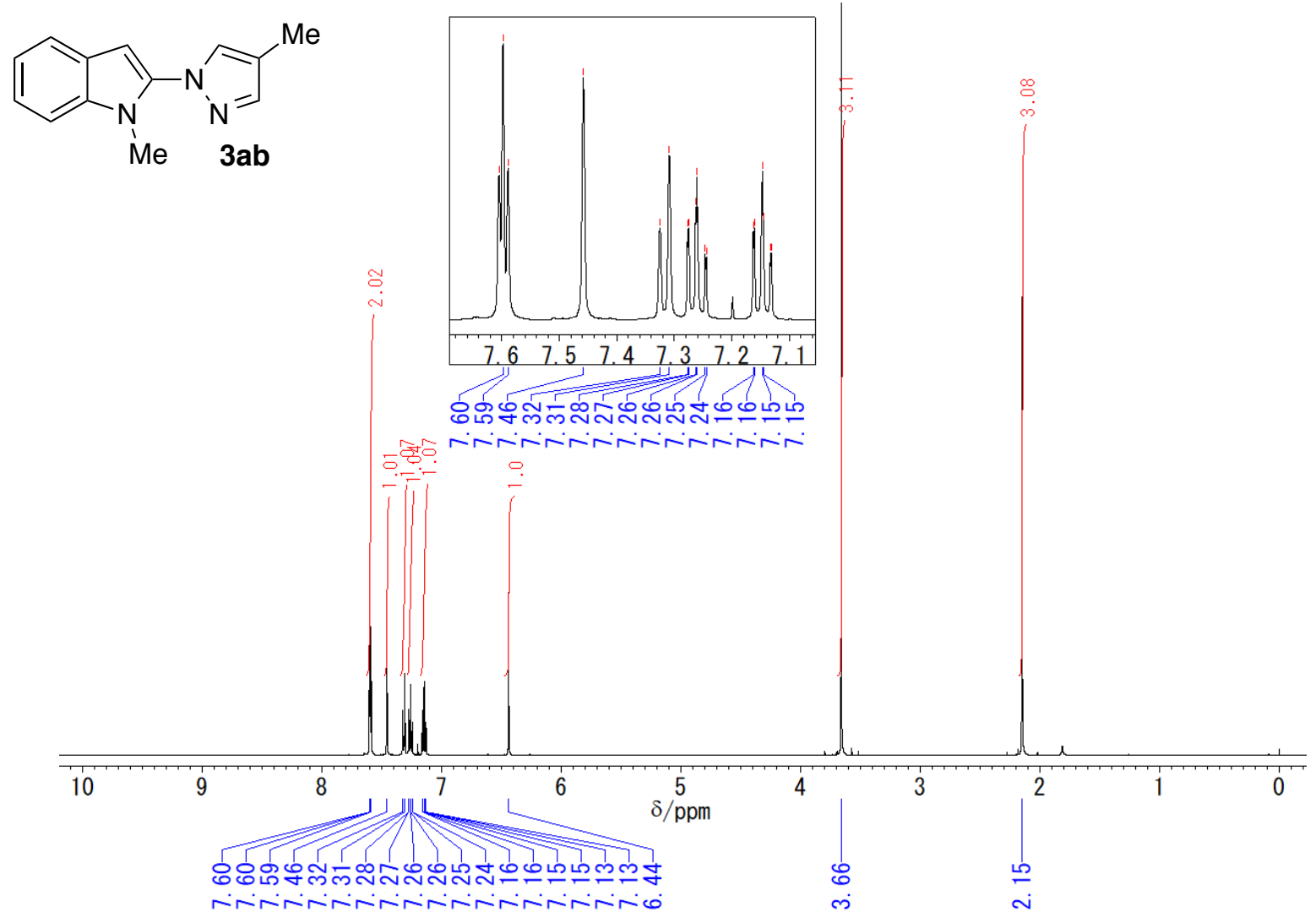

Spectrum S11. ${ }^{1} \mathrm{H} \mathrm{NMR}\left(\mathrm{CDCl}_{3}, 500 \mathrm{MHz}\right)$ spectrum of compound 3ab.

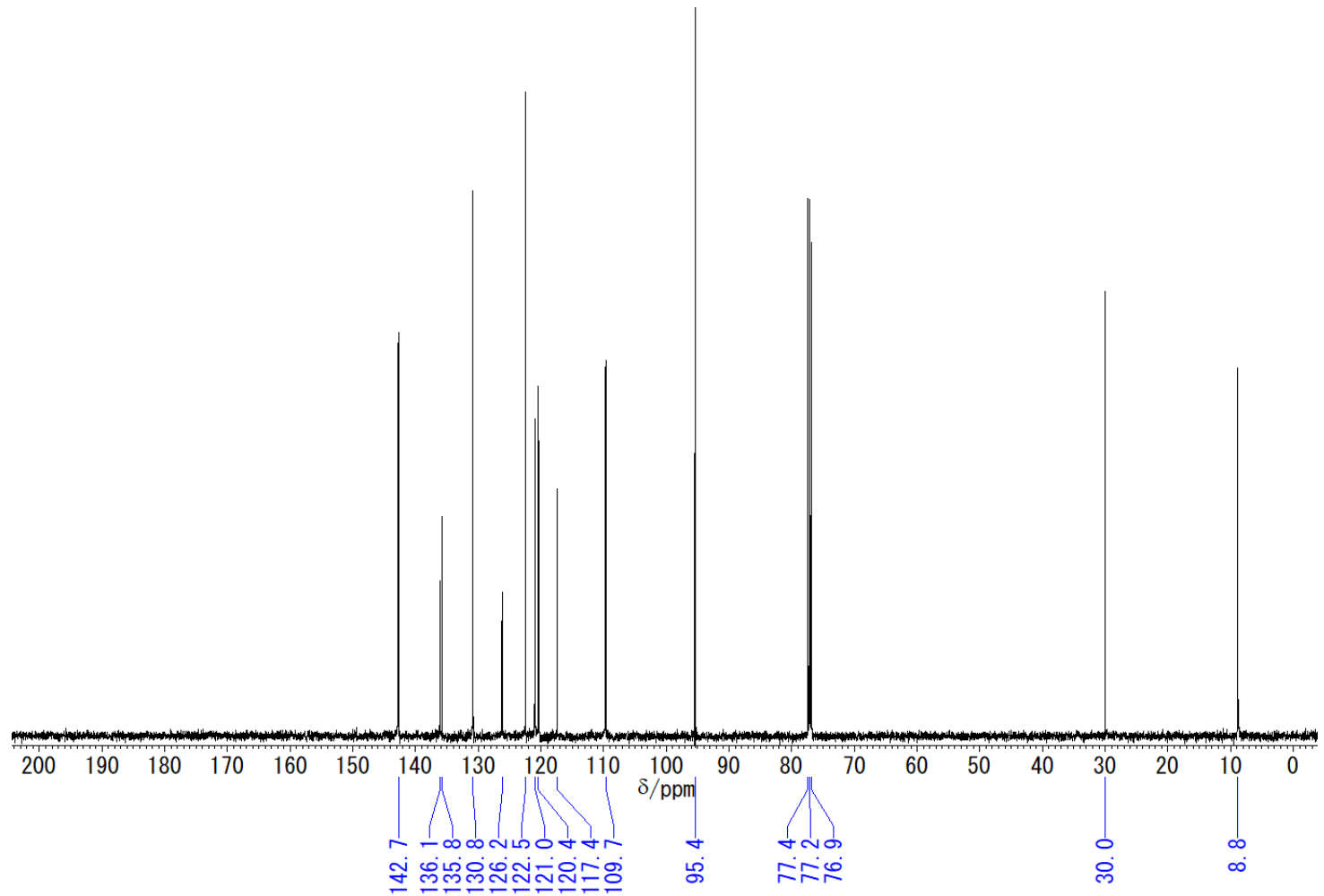

Spectrum S12. ${ }^{13} \mathrm{C}\left\{{ }^{1} \mathrm{H}\right\} \mathrm{NMR}\left(\mathrm{CDCl}_{3}, 126 \mathrm{MHz}\right)$ spectrum of compound 3ab. 


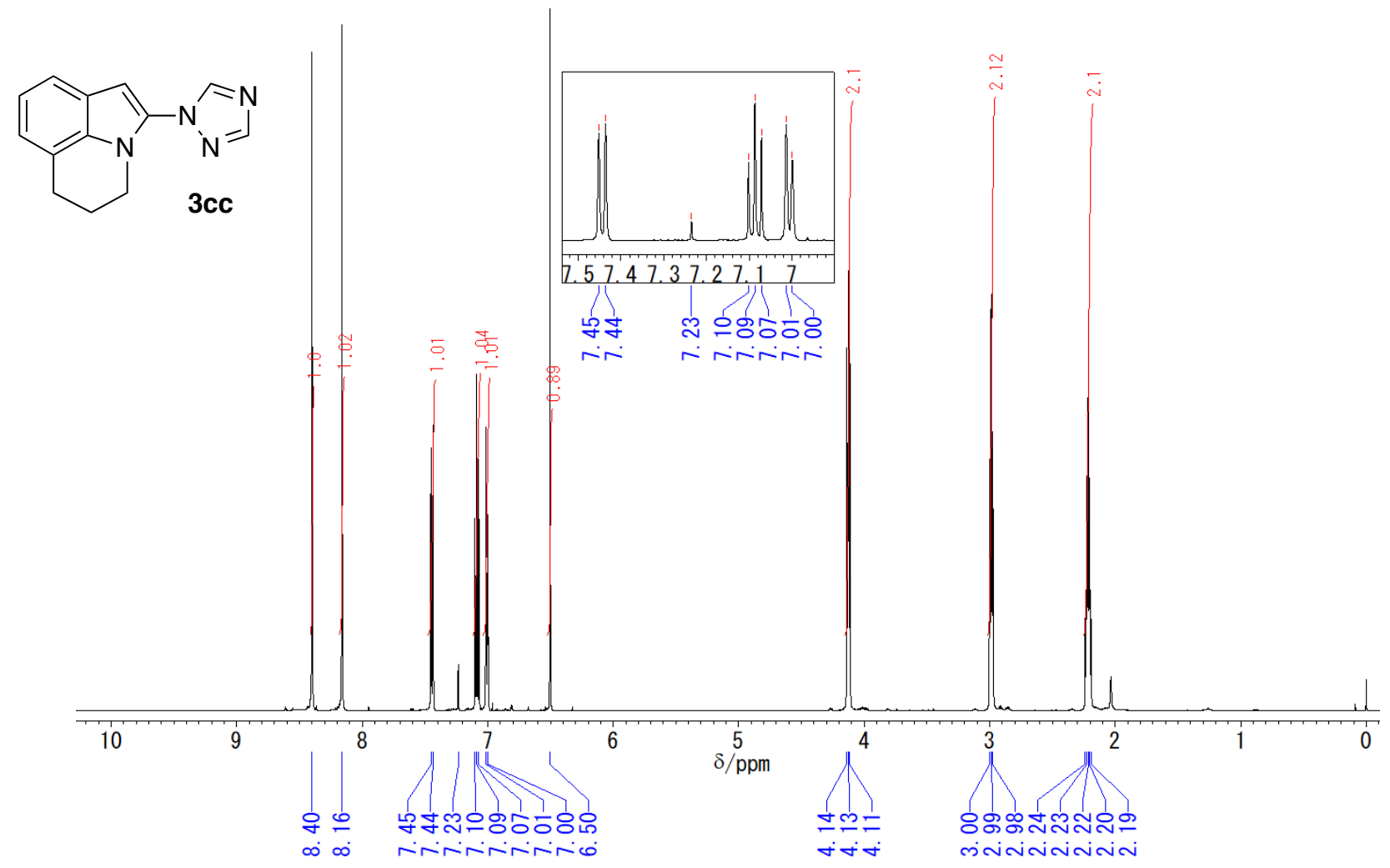

Spectrum S13. ${ }^{1} \mathrm{H}$ NMR $\left(\mathrm{CDCl}_{3}, 500 \mathrm{MHz}\right)$ spectrum of compound 3cc.

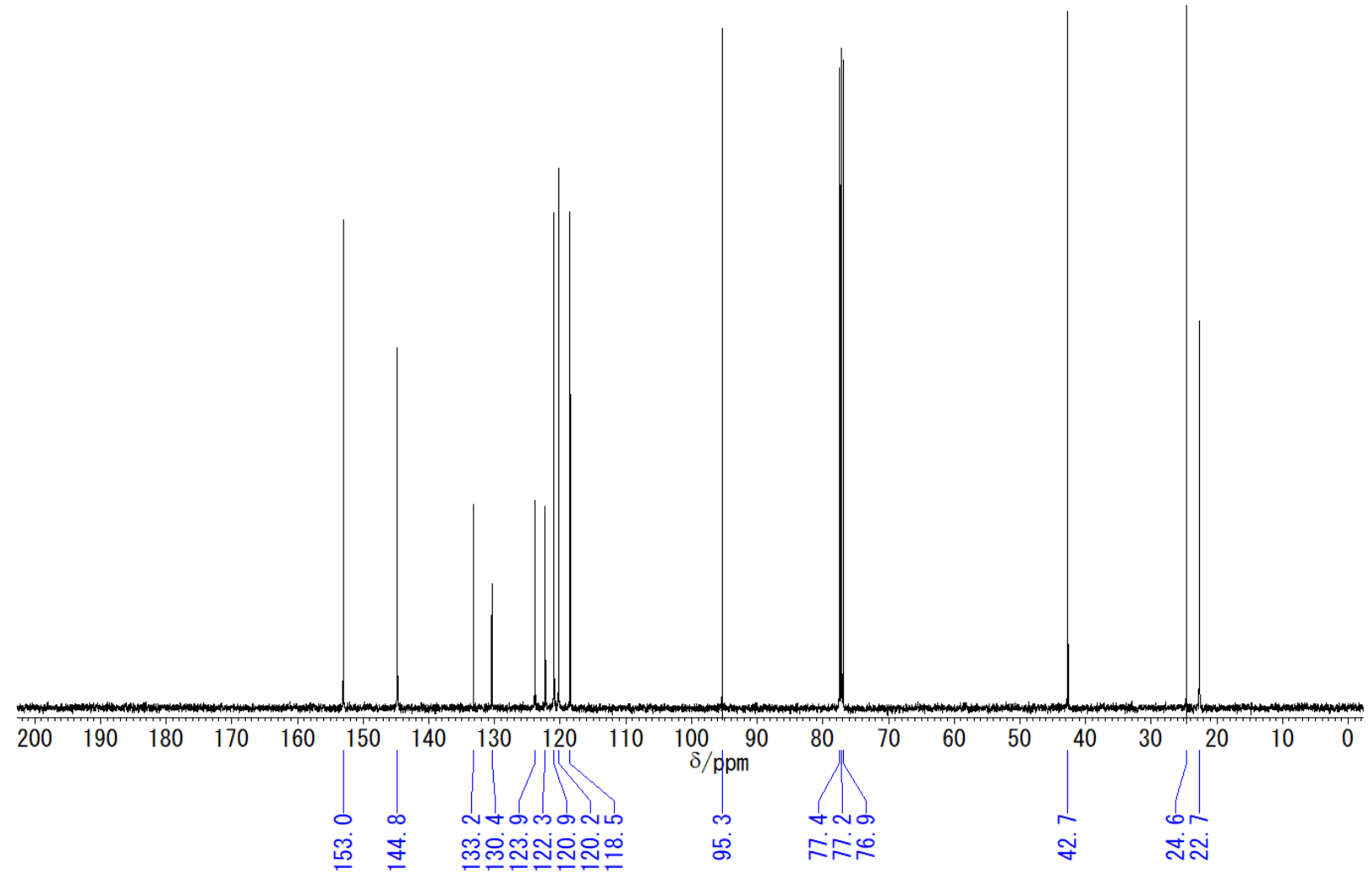

Spectrum S14. ${ }^{13} \mathrm{C}\left\{{ }^{1} \mathrm{H}\right\}$ NMR $\left(\mathrm{CDCl}_{3}, 126 \mathrm{MHz}\right)$ spectrum of compound 3cc. 
<smiles>Cc1ccc(Sc2c(-n3cccn3)n(C)c3ccccc23)cc1</smiles>
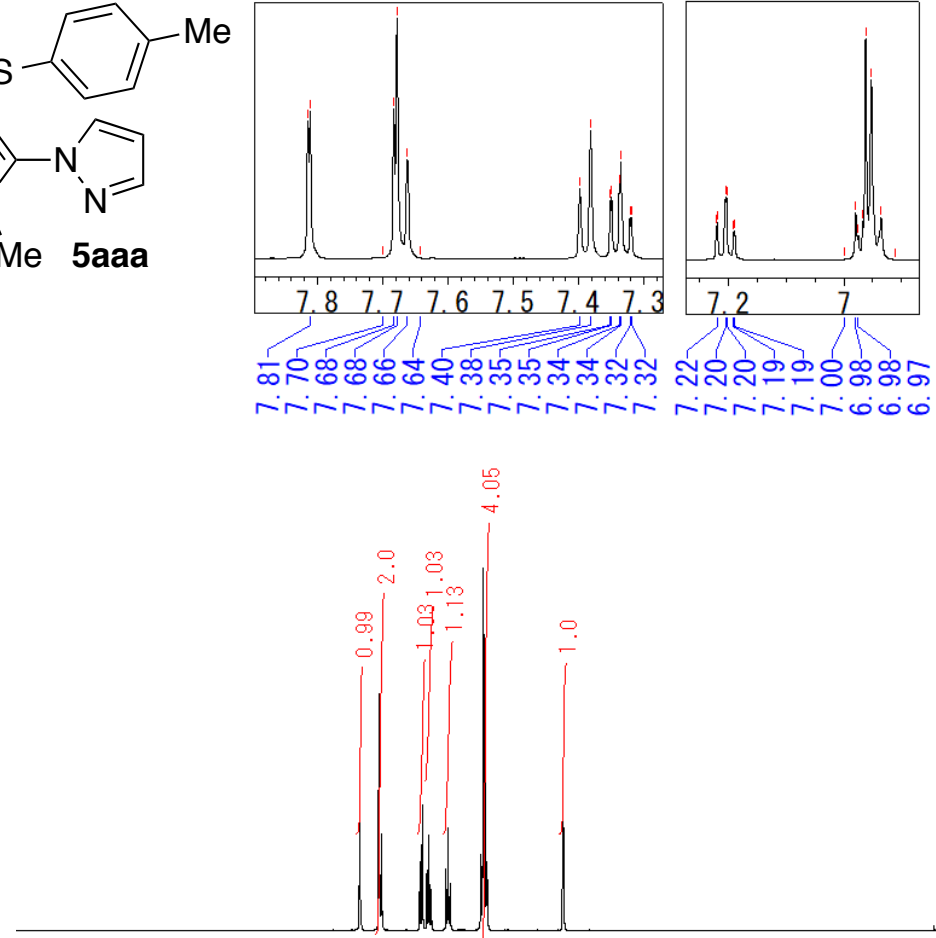

$\overline{10}$

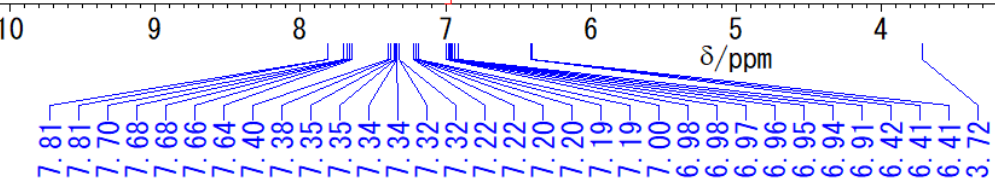

Spectrum S15. ${ }^{1} \mathrm{H}$ NMR $\left(\mathrm{CDCl}_{3}, 500 \mathrm{MHz}\right)$ spectrum of compound 5aaa.

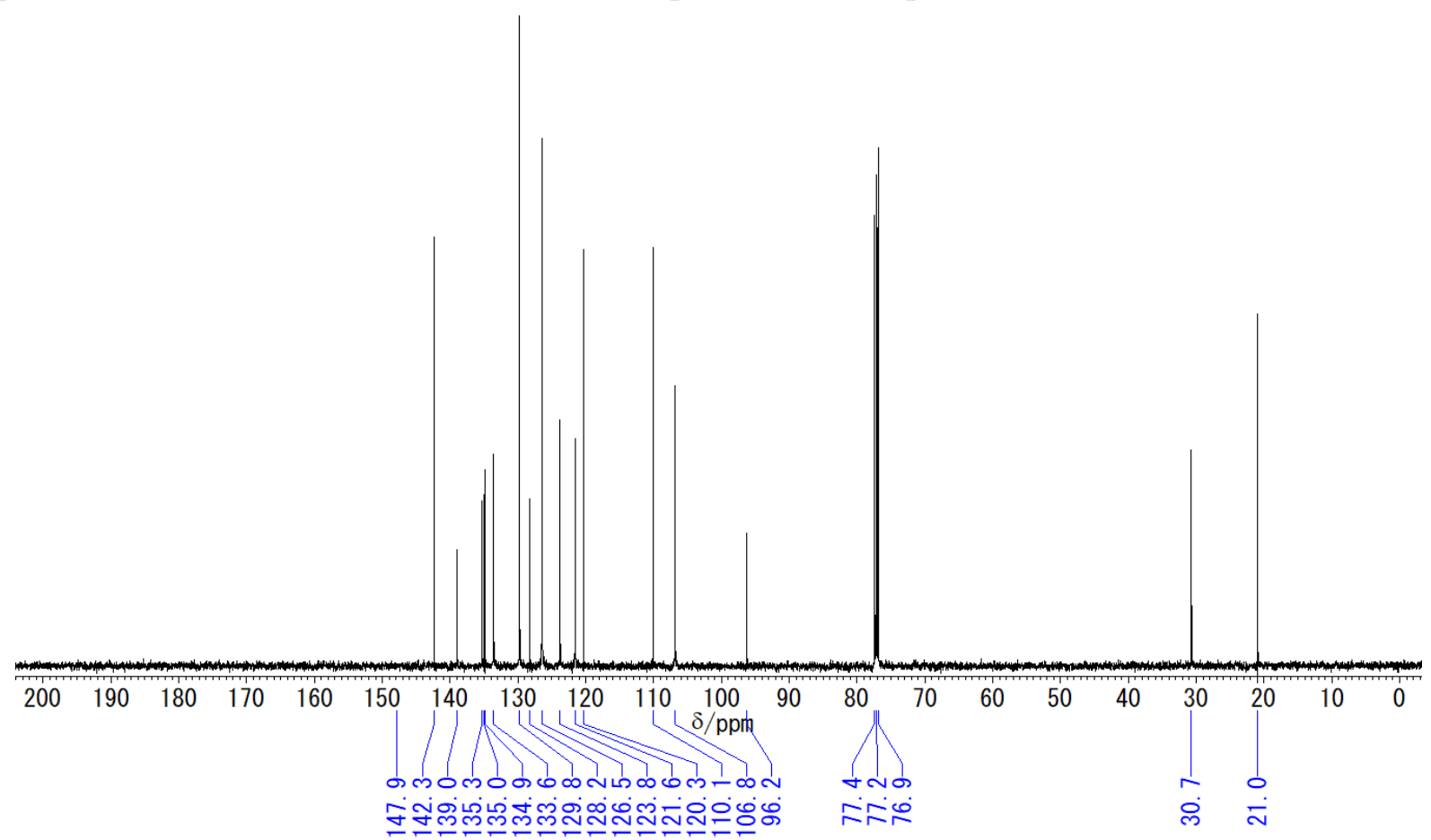

Spectrum S16. ${ }^{13} \mathrm{C}\left\{{ }^{1} \mathrm{H}\right\}$ NMR $\left(\mathrm{CDCl}_{3}, 126 \mathrm{MHz}\right)$ spectrum of compound 5 aaa. 


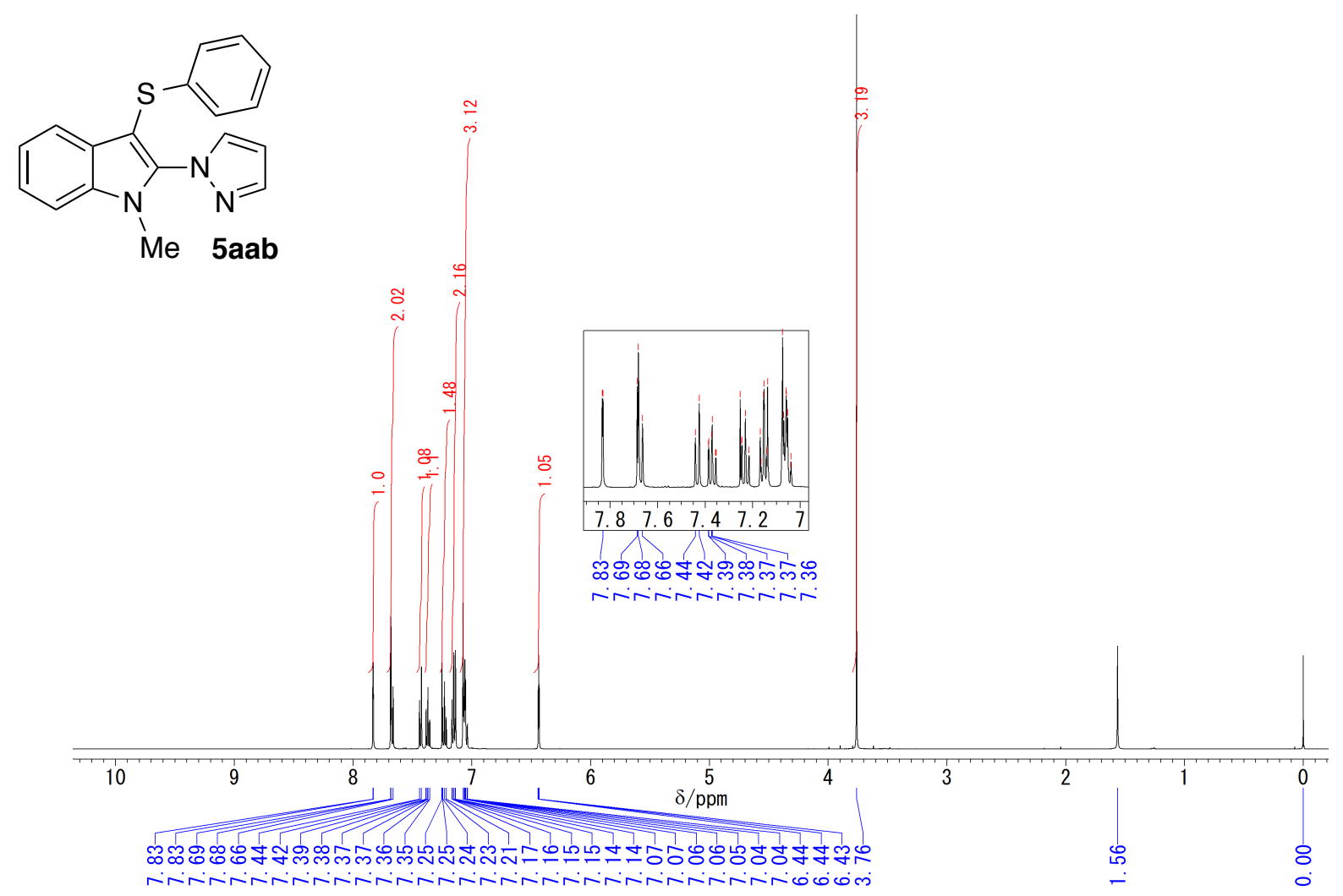

Spectrum S17. ${ }^{1} \mathrm{H} \mathrm{NMR}\left(\mathrm{CDCl}_{3}, 500 \mathrm{MHz}\right)$ spectrum of compound 5aab.

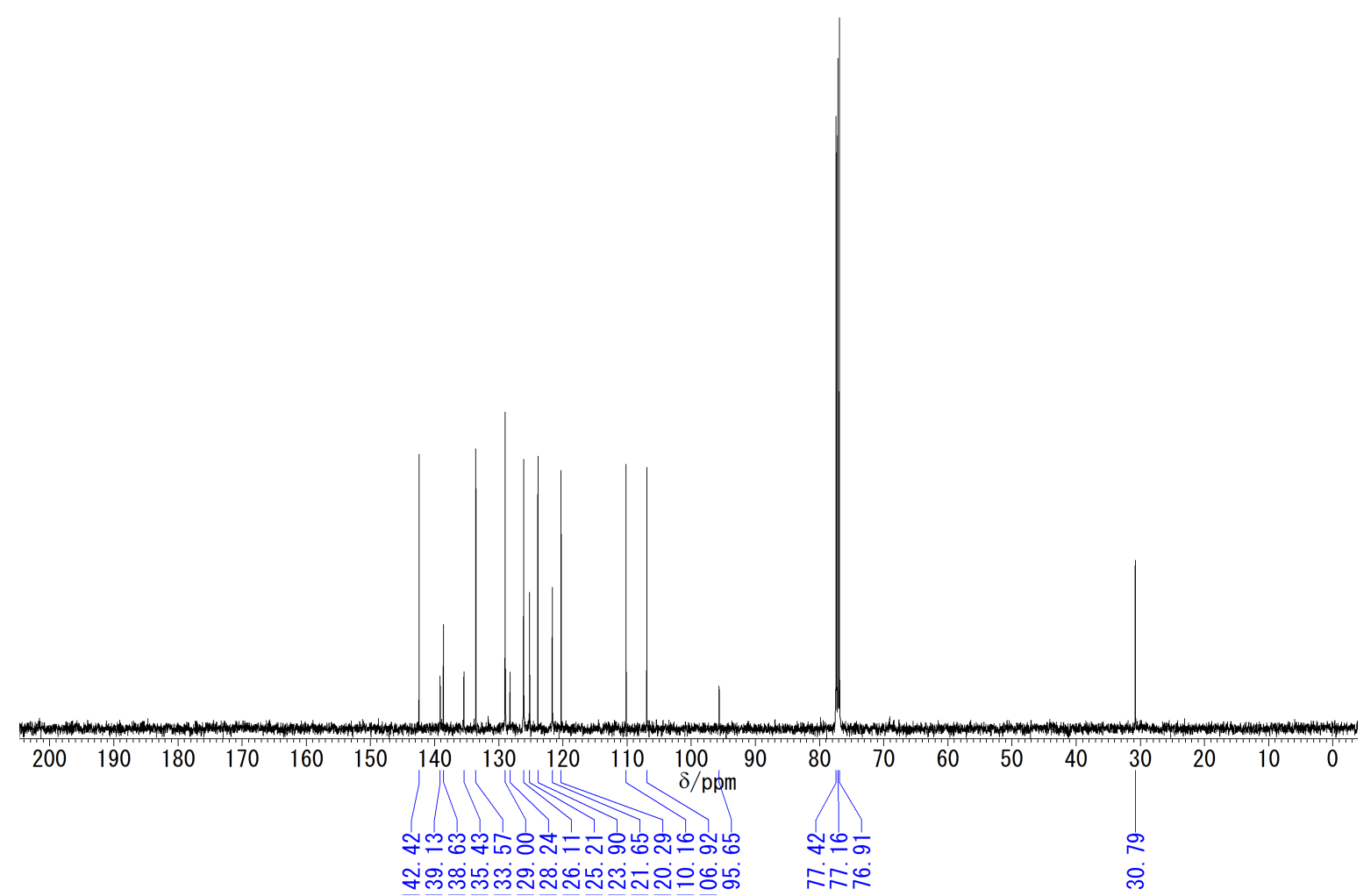

Spectrum S18. ${ }^{13} \mathrm{C}\left\{{ }^{1} \mathrm{H}\right\} \mathrm{NMR}\left(\mathrm{CDCl}_{3}, 126 \mathrm{MHz}\right)$ spectrum of compound 5aab. 
<smiles>CSc1c(-n2cccn2)n(C)c2ccccc12</smiles>
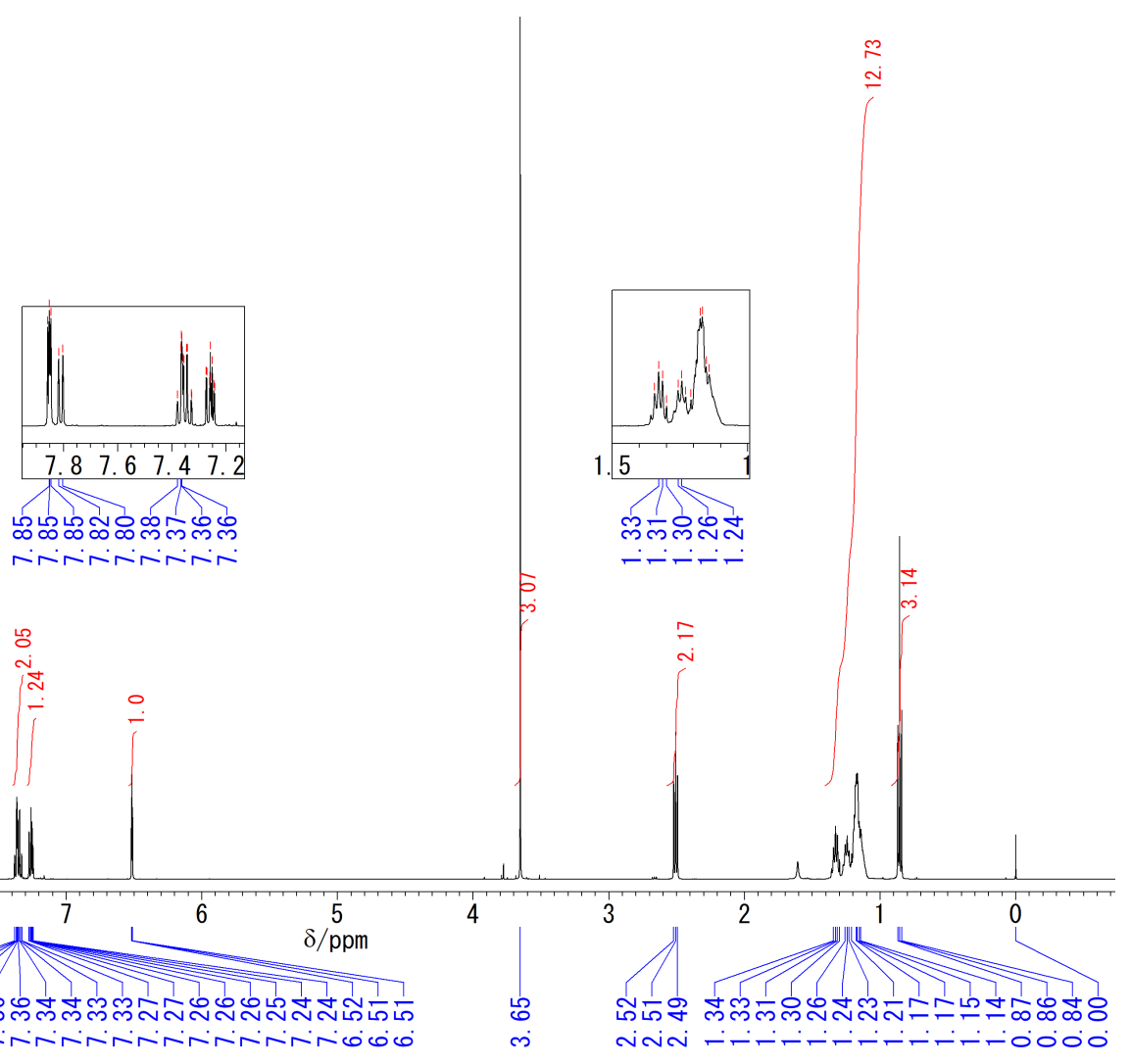

Spectrum S19. ${ }^{1} \mathrm{H} \mathrm{NMR}\left(\mathrm{CDCl}_{3}, 500 \mathrm{MHz}\right)$ spectrum of compound 5aac.

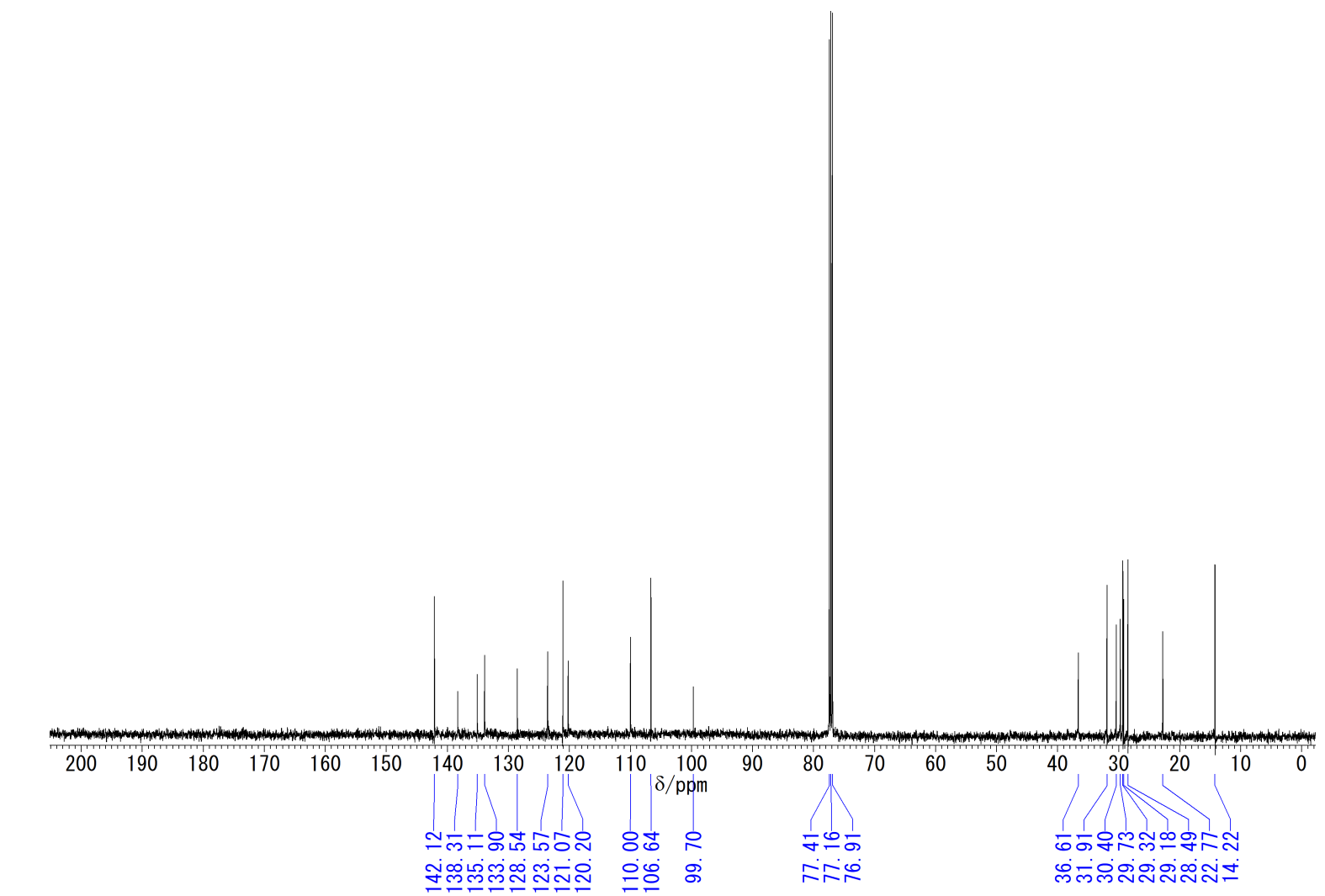

Spectrum S20. ${ }^{13} \mathrm{C}\left\{{ }^{1} \mathrm{H}\right\} \mathrm{NMR}\left(\mathrm{CDCl}_{3}, 126 \mathrm{MHz}\right)$ spectrum of compound 5aac. 


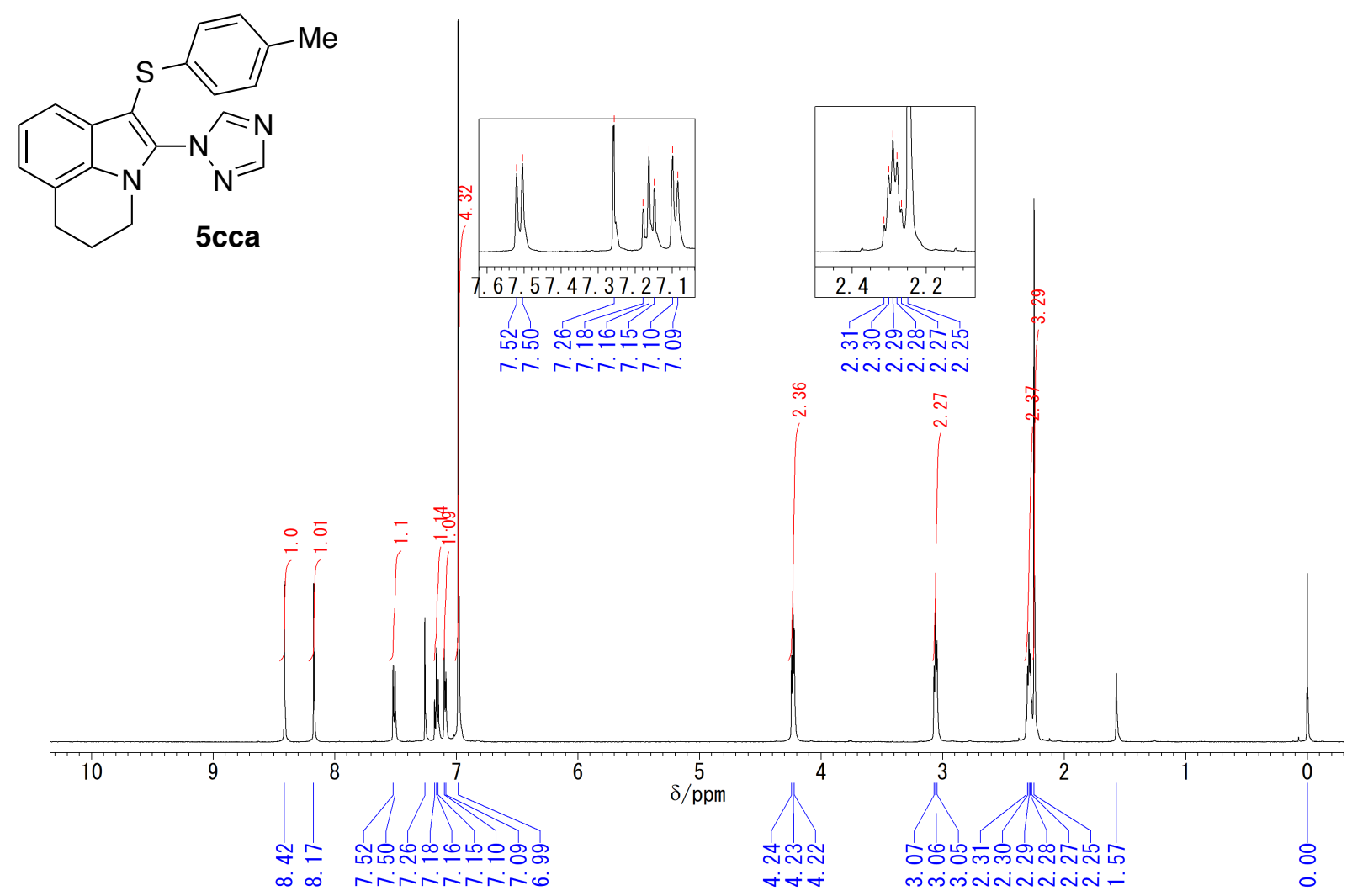

Spectrum S21. ${ }^{1} \mathrm{H}$ NMR $\left(\mathrm{CDCl}_{3}, 500 \mathrm{MHz}\right)$ spectrum of compound $\mathbf{5 c c a}$.

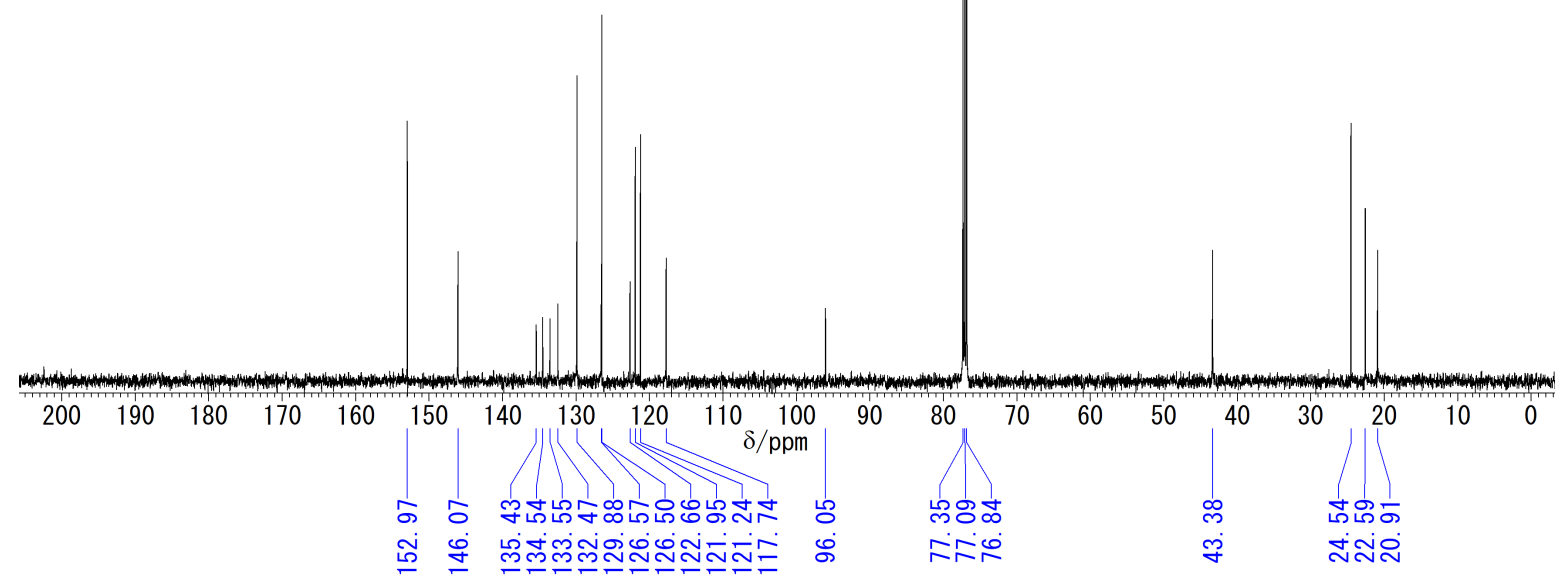

Spectrum S22. ${ }^{13} \mathrm{C}\left\{{ }^{1} \mathrm{H}\right\}$ NMR $\left(\mathrm{CDCl}_{3}, 126 \mathrm{MHz}\right)$ spectrum of compound $\mathbf{5 c c a}$. 


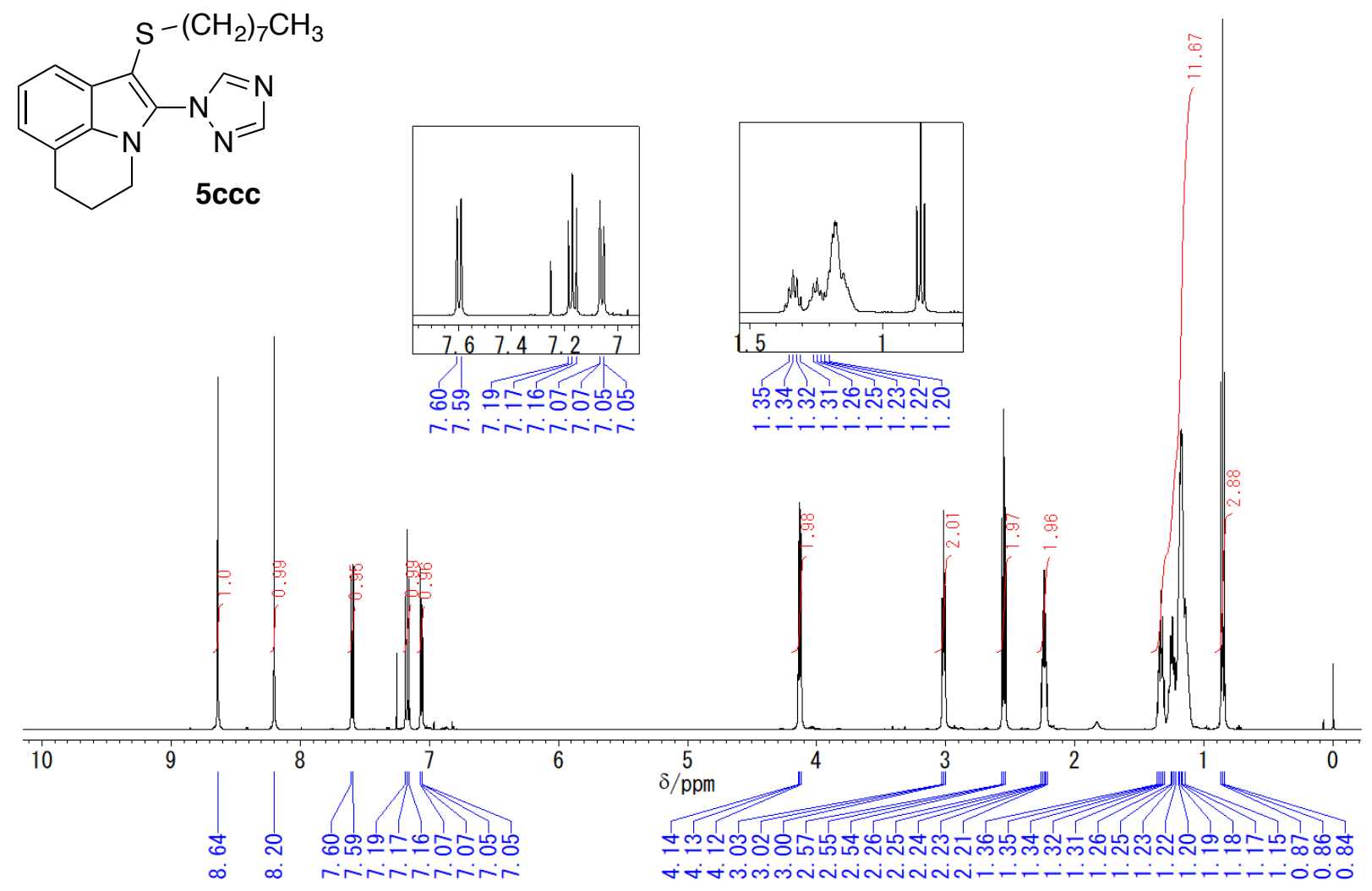

Spectrum S23. ${ }^{1} \mathrm{H}$ NMR $\left(\mathrm{CDCl}_{3}, 500 \mathrm{MHz}\right)$ spectrum of compound 5cce.

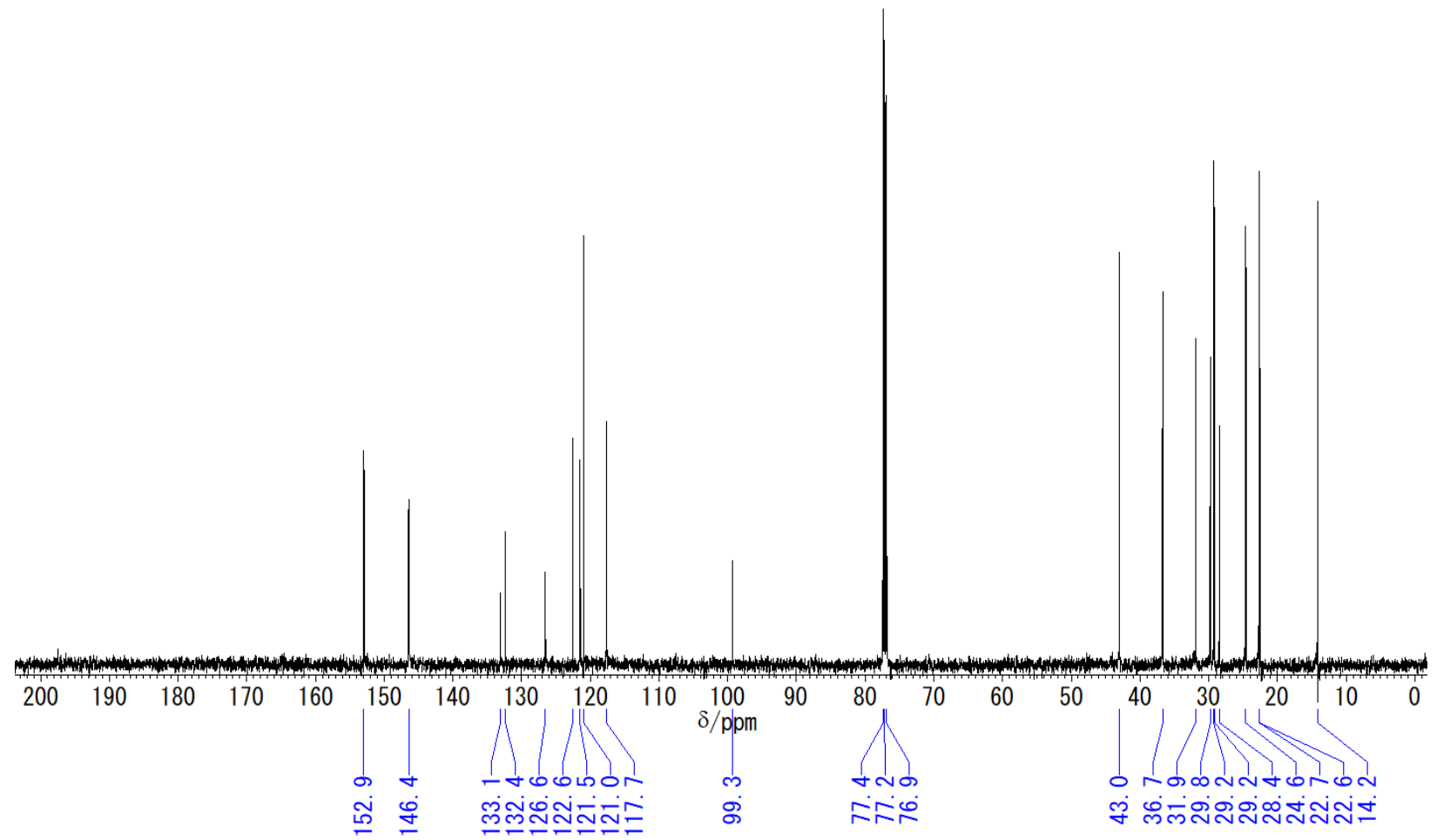

Spectrum S24. ${ }^{13} \mathrm{C}\left\{{ }^{1} \mathrm{H}\right\}$ NMR $\left(\mathrm{CDCl}_{3}, 126 \mathrm{MHz}\right)$ spectrum of compound 5ccc. 


\section{5. ${ }^{1} \mathrm{H}$ and ${ }^{13} \mathrm{C}\left\{{ }^{1} \mathrm{H}\right\}$ NMR Spectra of Known Compounds}<smiles>Cn1c(-n2cccn2)cc2ccccc21</smiles>
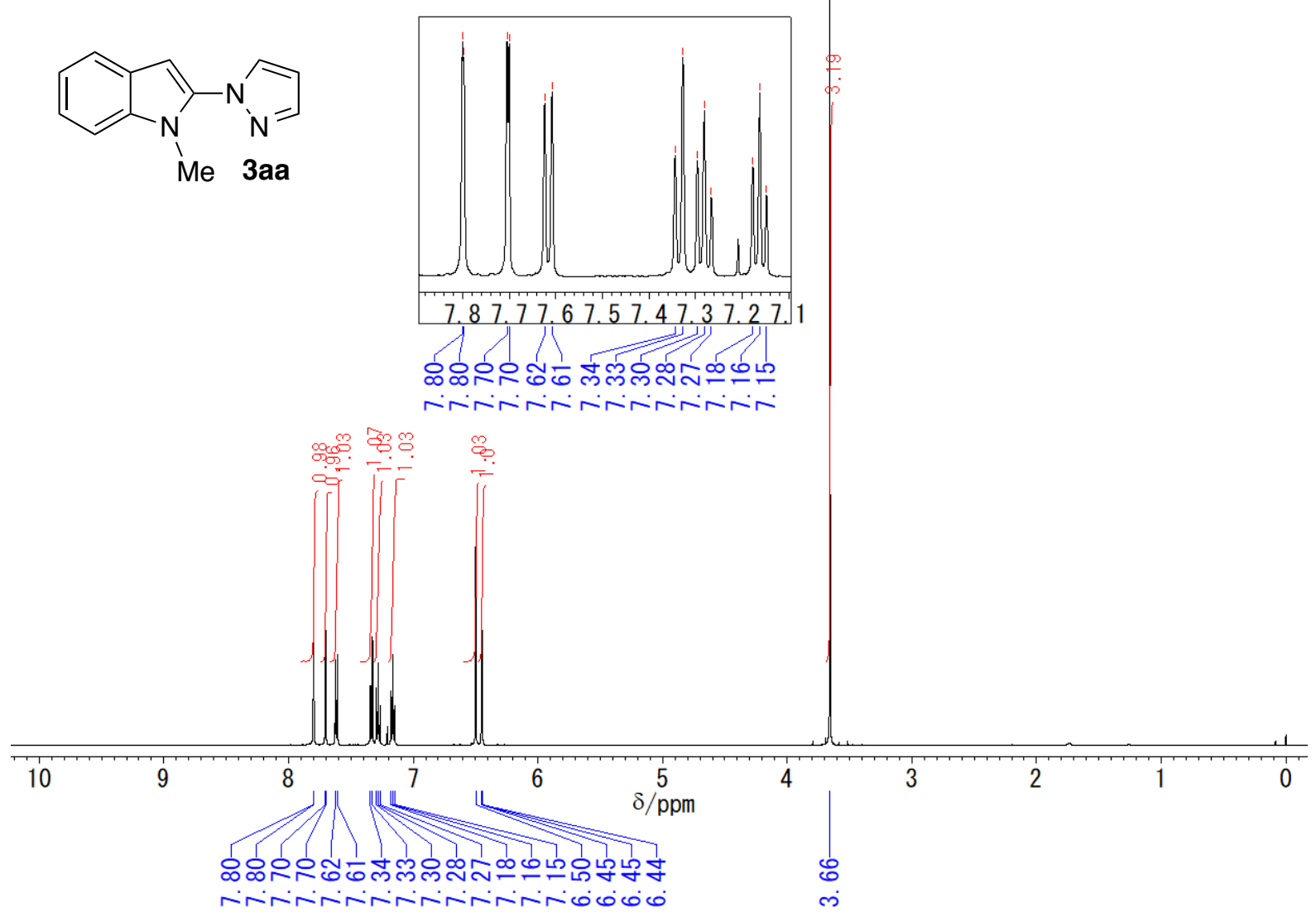

Spectrum S25. ${ }^{1} \mathrm{H}$ NMR $\left(\mathrm{CDCl}_{3}, 500 \mathrm{MHz}\right)$ spectrum of compound 3aa.

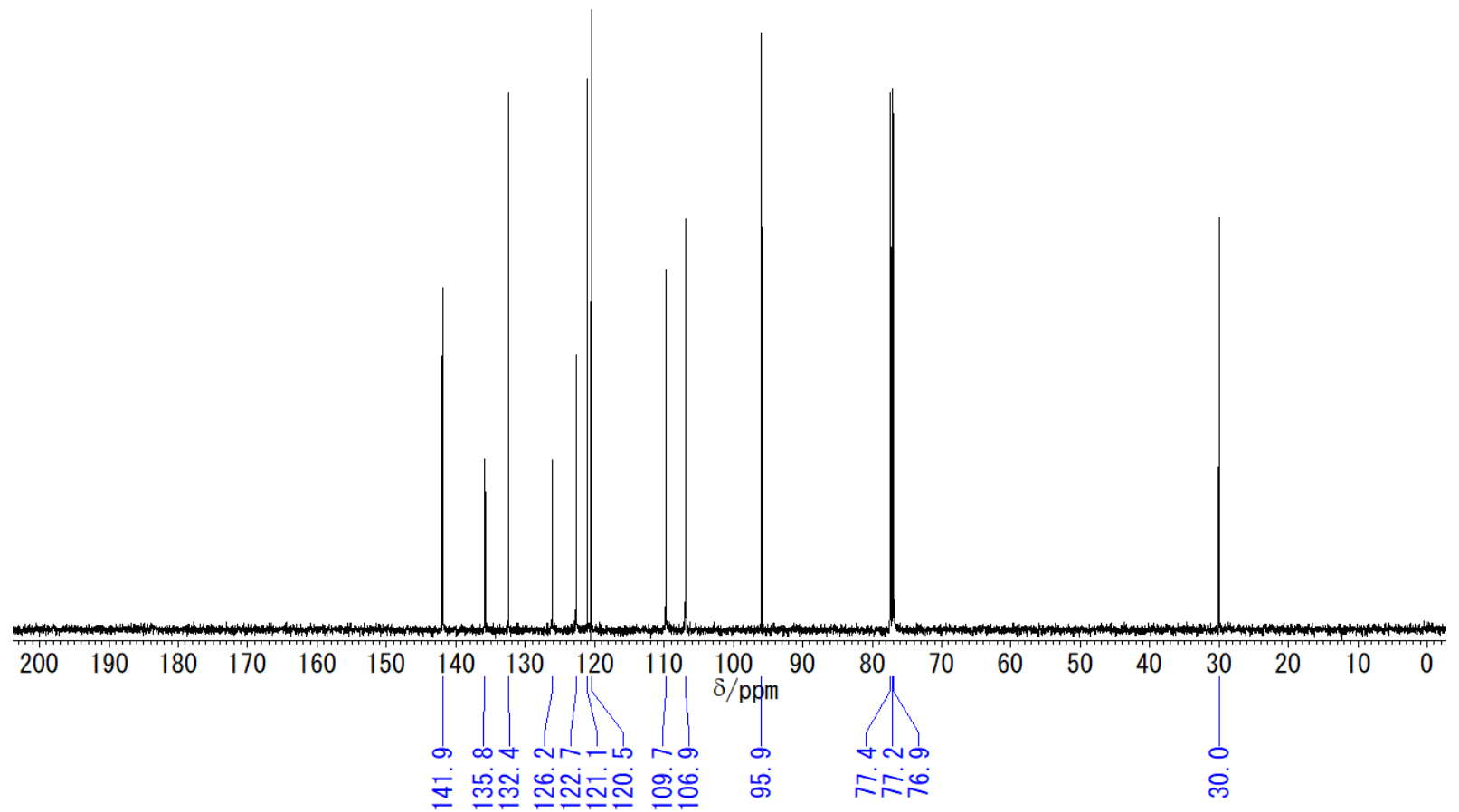

Spectrum S26. ${ }^{13} \mathrm{C}\left\{{ }^{1} \mathrm{H}\right\}$ NMR $\left(\mathrm{CDCl}_{3}, 126 \mathrm{MHz}\right)$ spectrum of compound 3aa. 
<smiles>Cc1c(-n2cccn2)[nH]c2ccccc12</smiles>

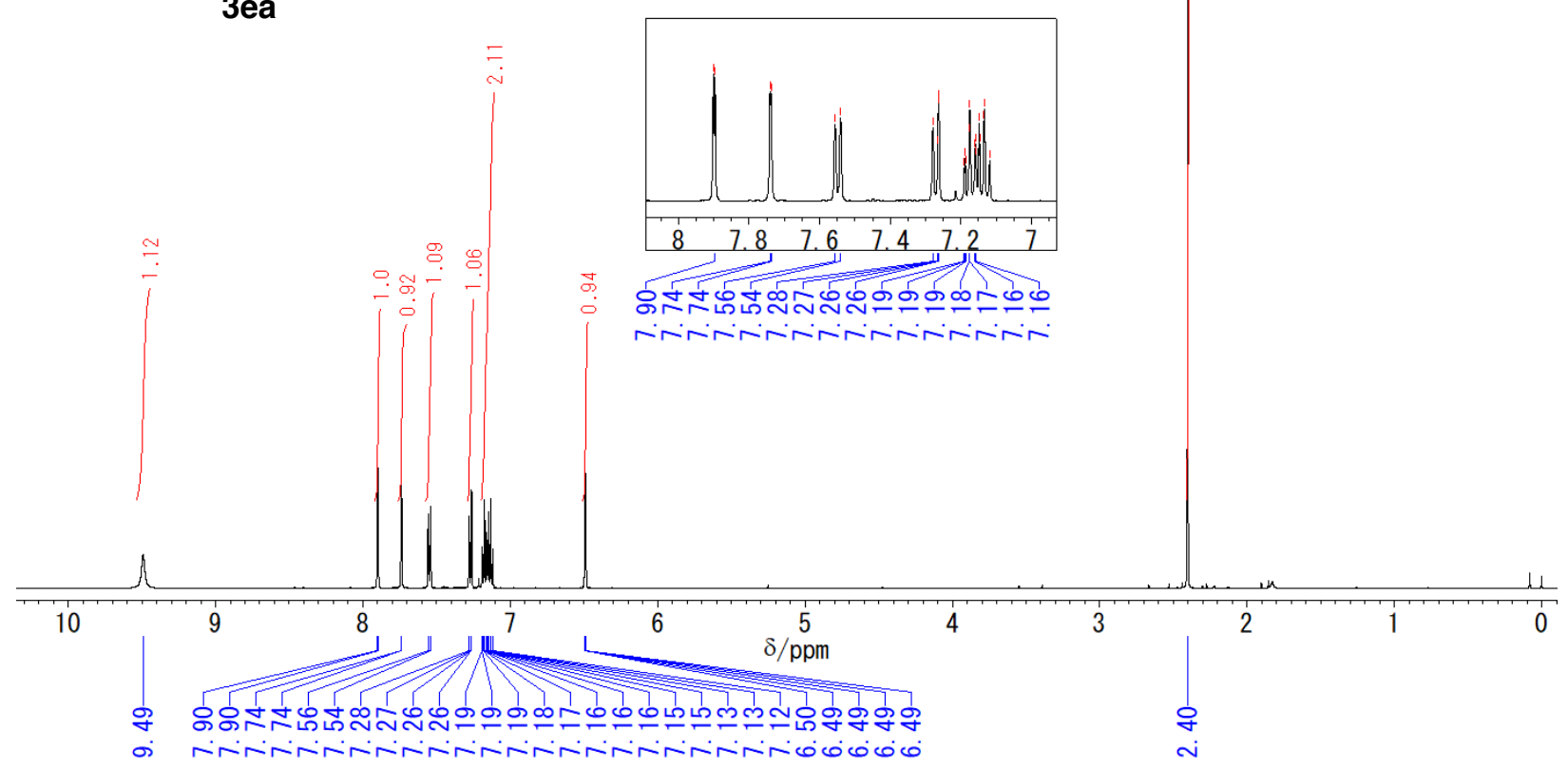

Spectrum S27. ${ }^{1} \mathrm{H} \mathrm{NMR}\left(\mathrm{CDCl}_{3}, 500 \mathrm{MHz}\right)$ spectrum of compound 3ea.

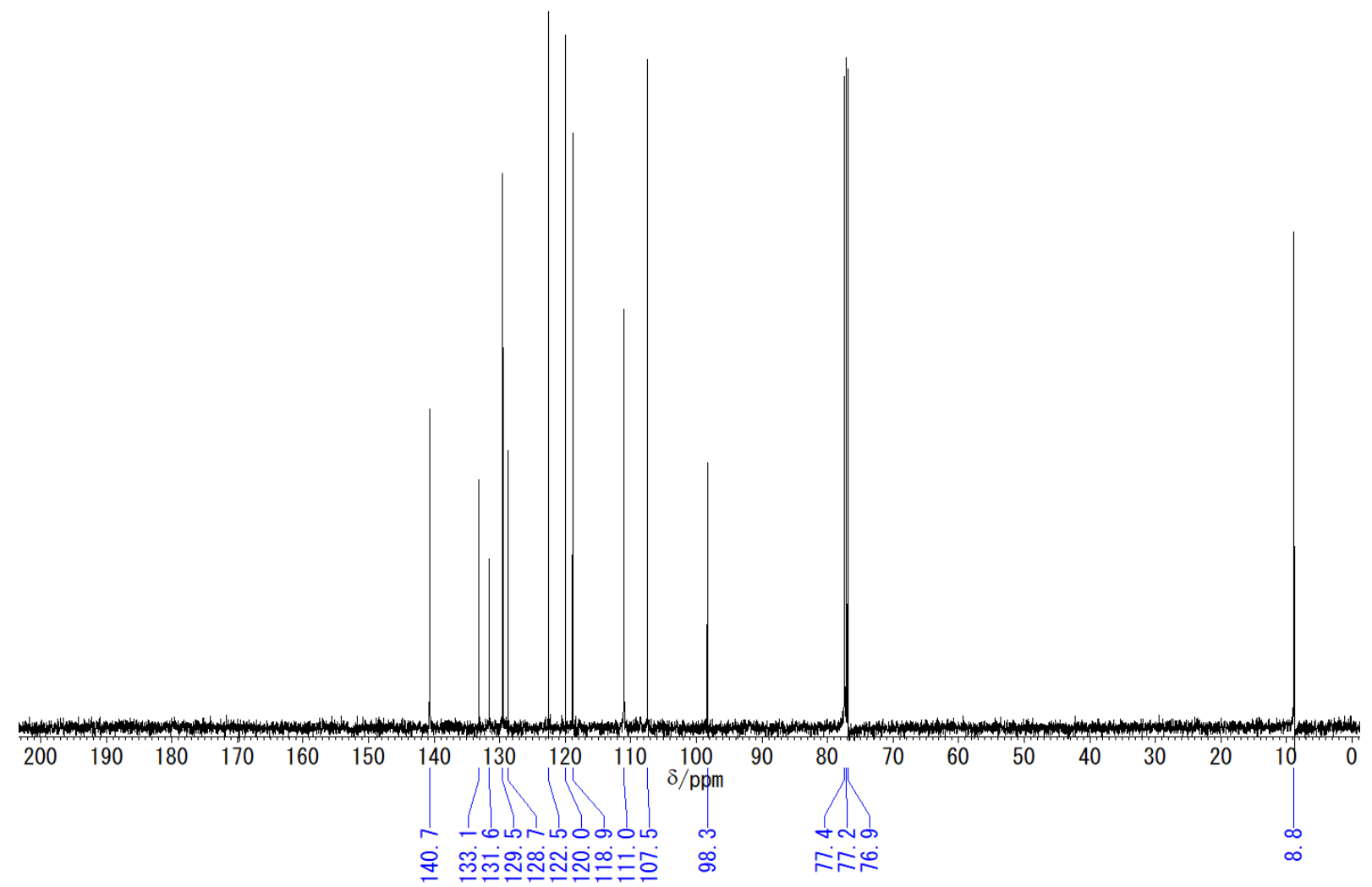

Spectrum S28. ${ }^{13} \mathrm{C}\left\{{ }^{1} \mathrm{H}\right\}$ NMR $\left(\mathrm{CDCl}_{3} 126 \mathrm{MHz}\right)$ spectrum of compound 3ea. 


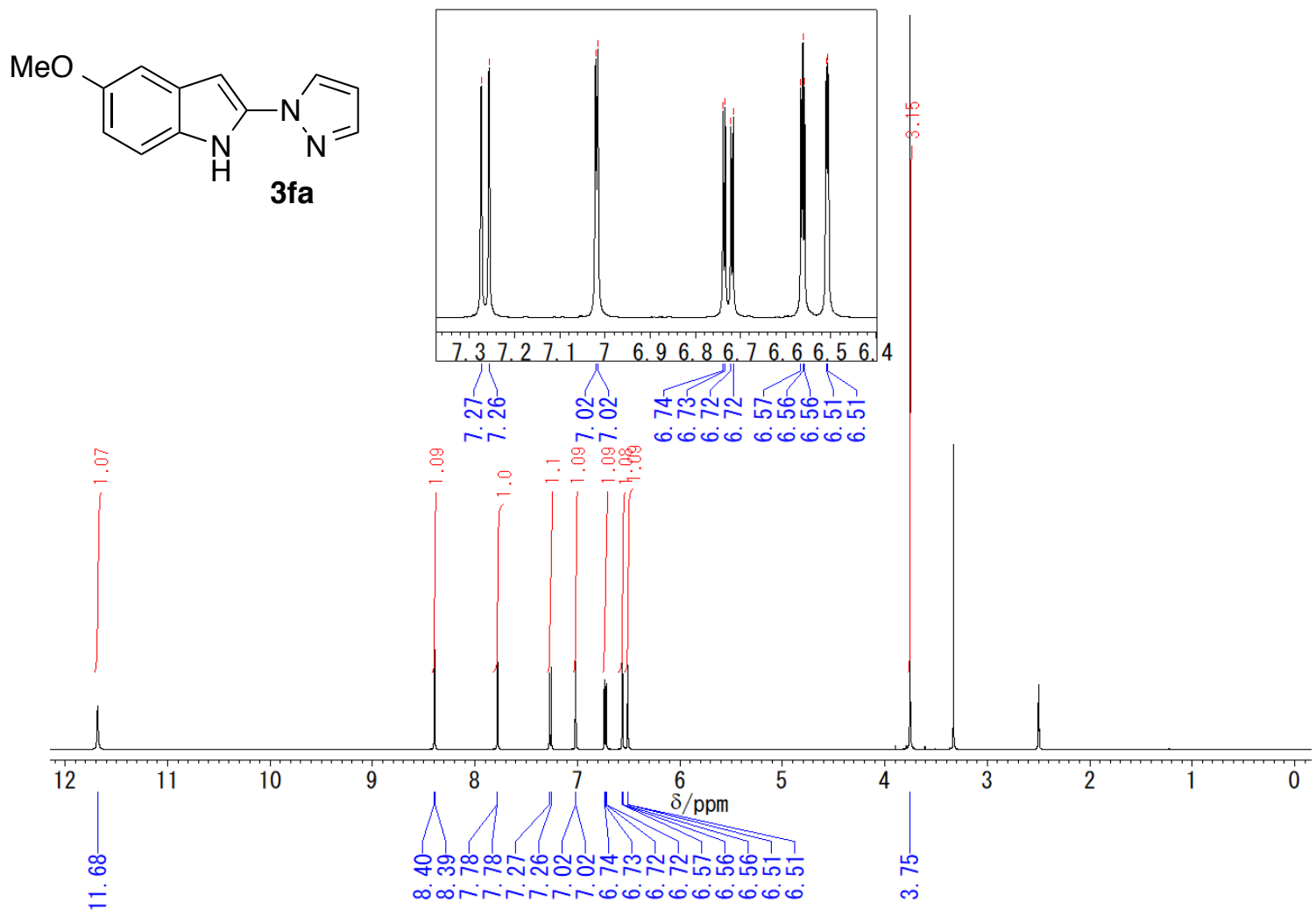

Spectrum S29. ${ }^{1} \mathrm{H}$ NMR (DMSO- $d_{6}, 500 \mathrm{MHz}$ ) spectrum of compound $\mathbf{3 f a}$.

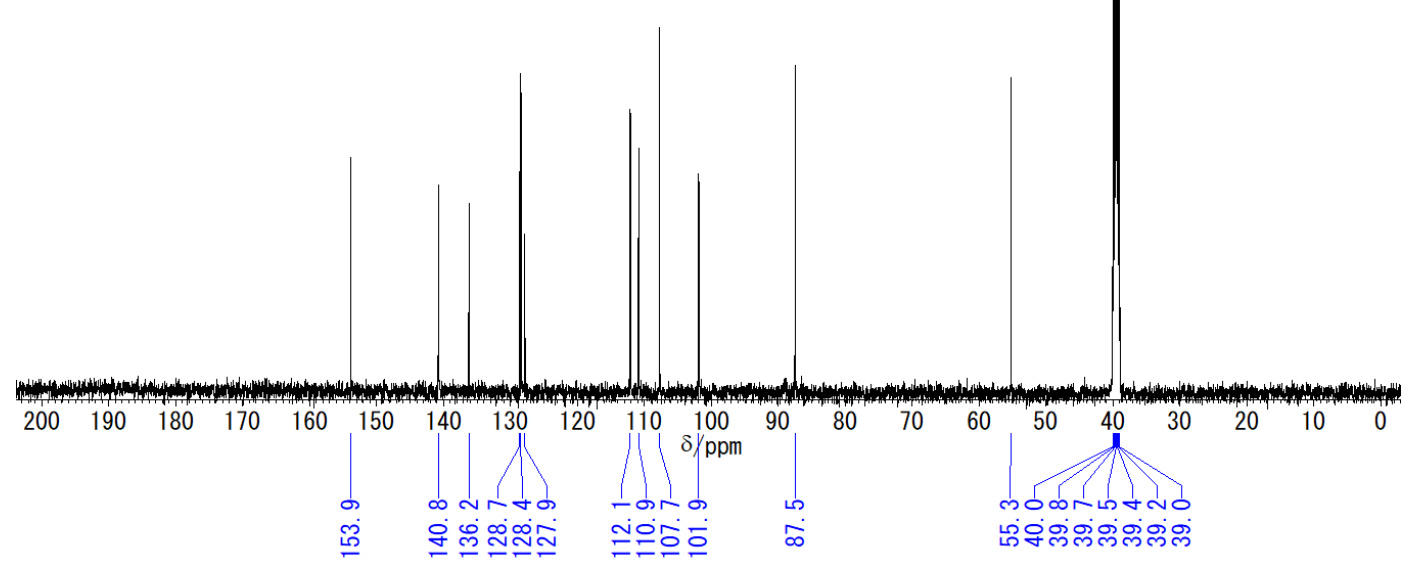

Spectrum S30. ${ }^{13} \mathrm{C}\left\{{ }^{1} \mathrm{H}\right\}$ NMR (DMSO- $d_{6}, 126 \mathrm{MHz}$ ) spectrum of compound 3 fa. 


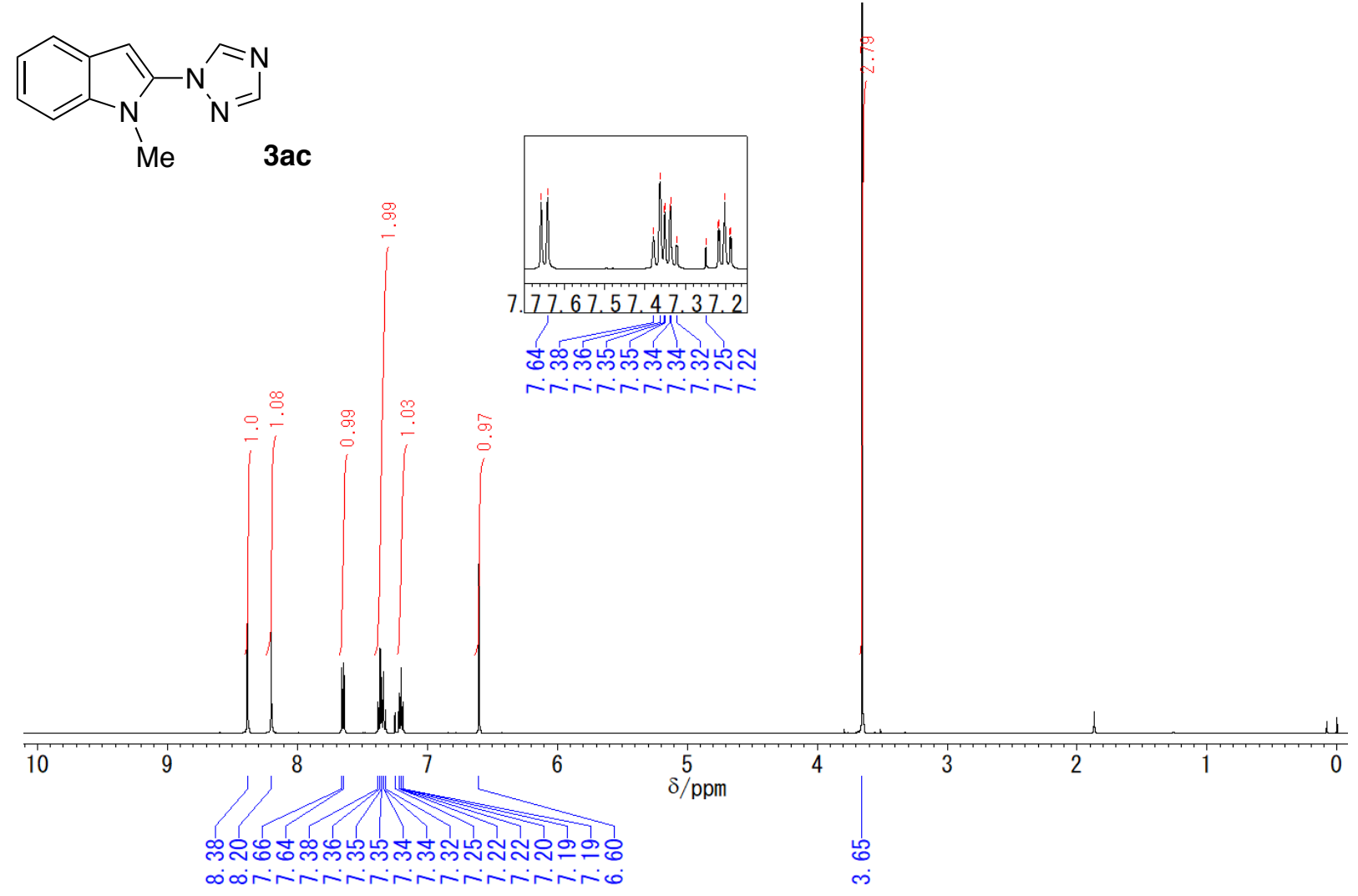

Spectrum S31. ${ }^{1} \mathrm{H} \mathrm{NMR}\left(\mathrm{CDCl}_{3}, 500 \mathrm{MHz}\right)$ spectrum of compound 3ac.

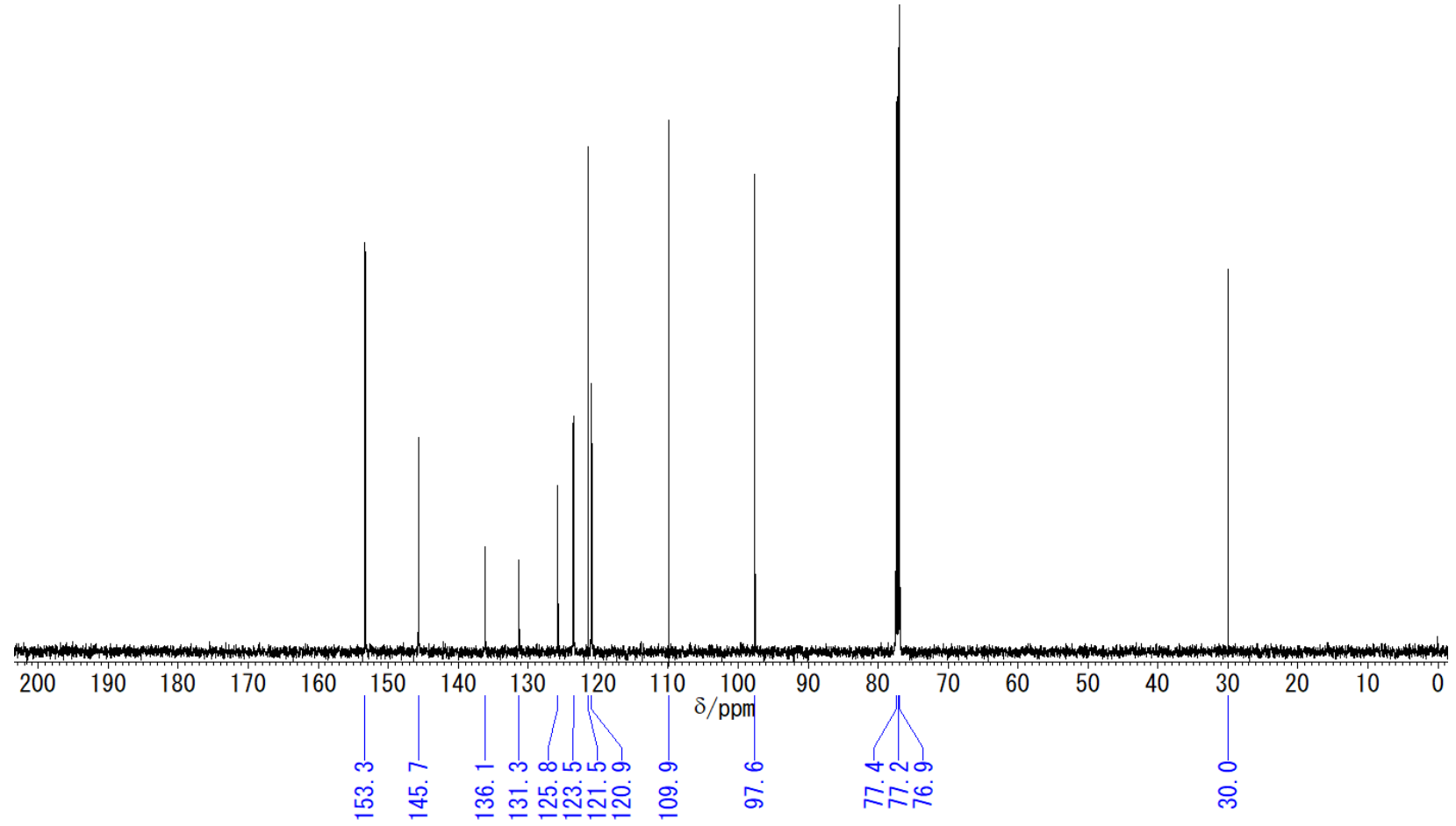

Spectrum S32. ${ }^{13} \mathrm{C}\left\{{ }^{1} \mathrm{H}\right\}$ NMR $\left(\mathrm{CDCl}_{3} 126 \mathrm{MHz}\right)$ spectrum of compound 3ac. 


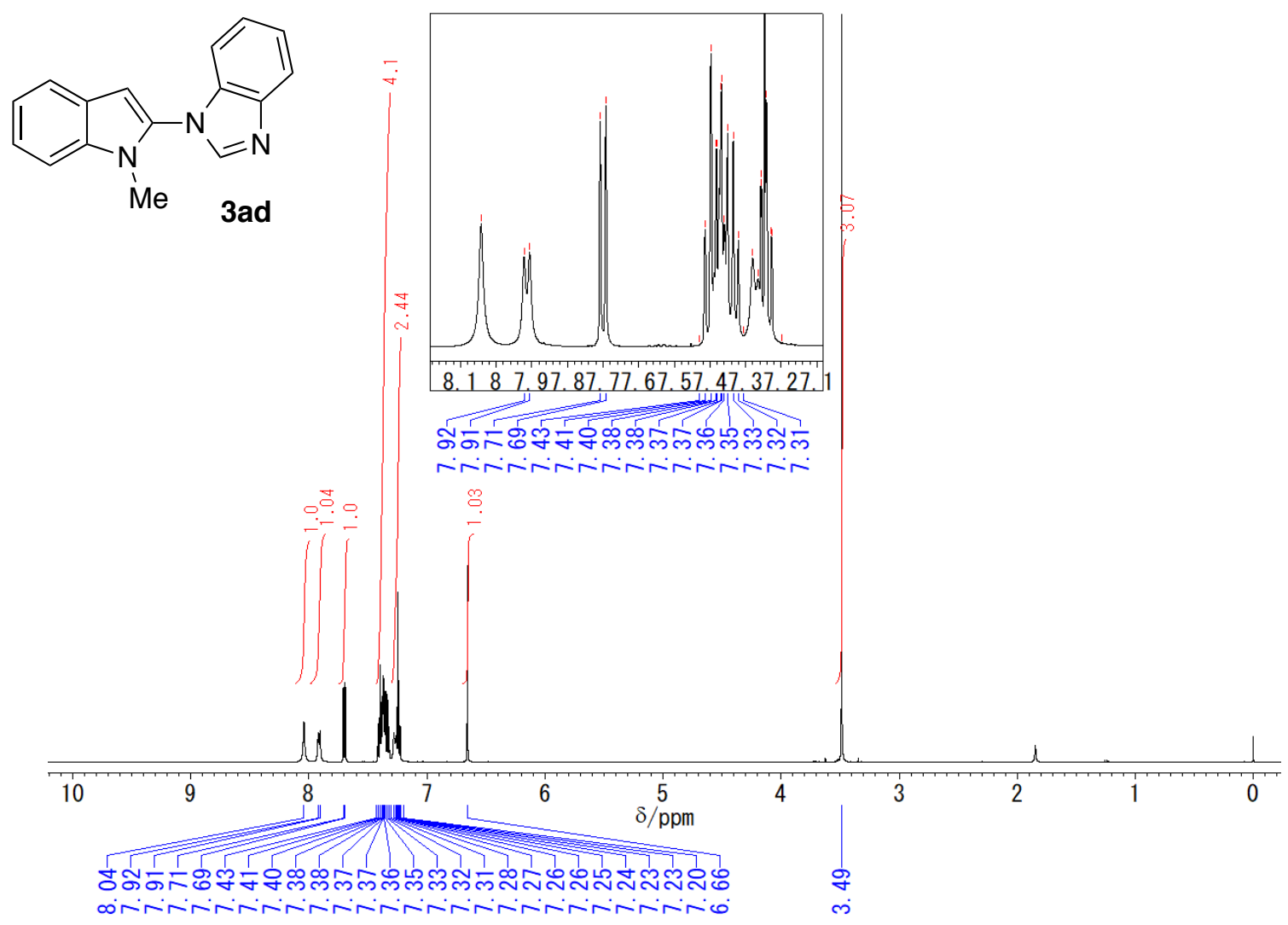

Spectrum S33. ${ }^{1} \mathrm{H}$ NMR $\left(\mathrm{CDCl}_{3}, 500 \mathrm{MHz}\right)$ spectrum of compound 3 ad.

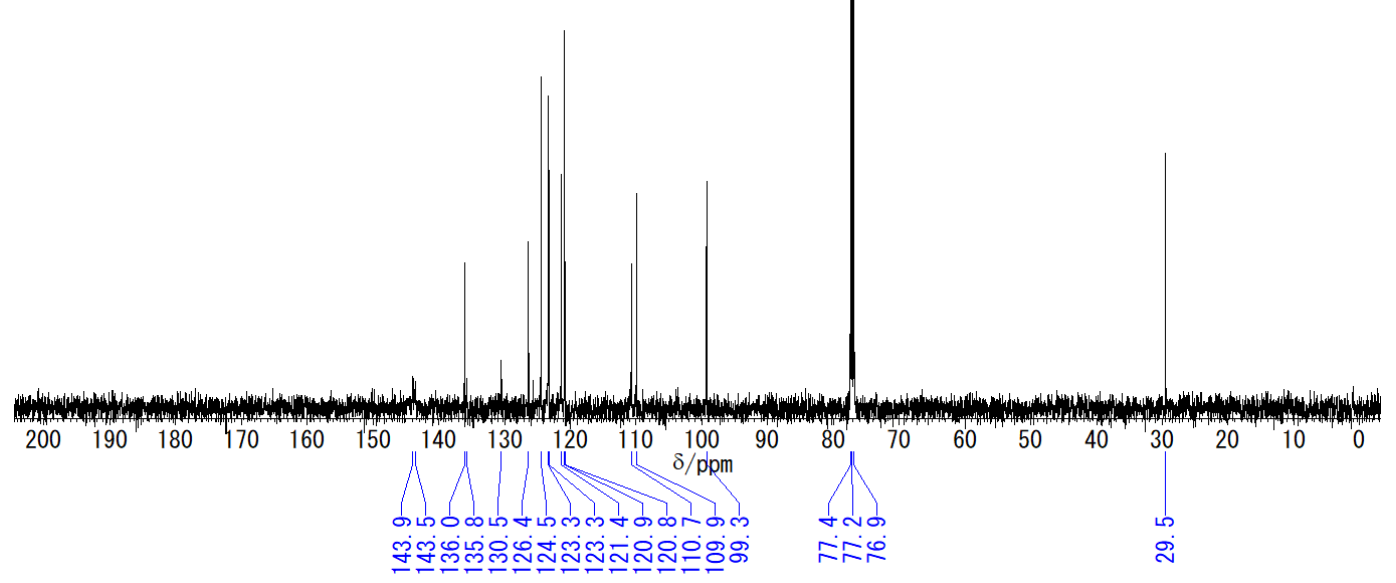

Spectrum S34. ${ }^{13} \mathrm{C}\left\{{ }^{1} \mathrm{H}\right\}$ NMR $\left(\mathrm{CDCl}_{3}, 126 \mathrm{MHz}\right)$ spectrum of compound 3ad. 


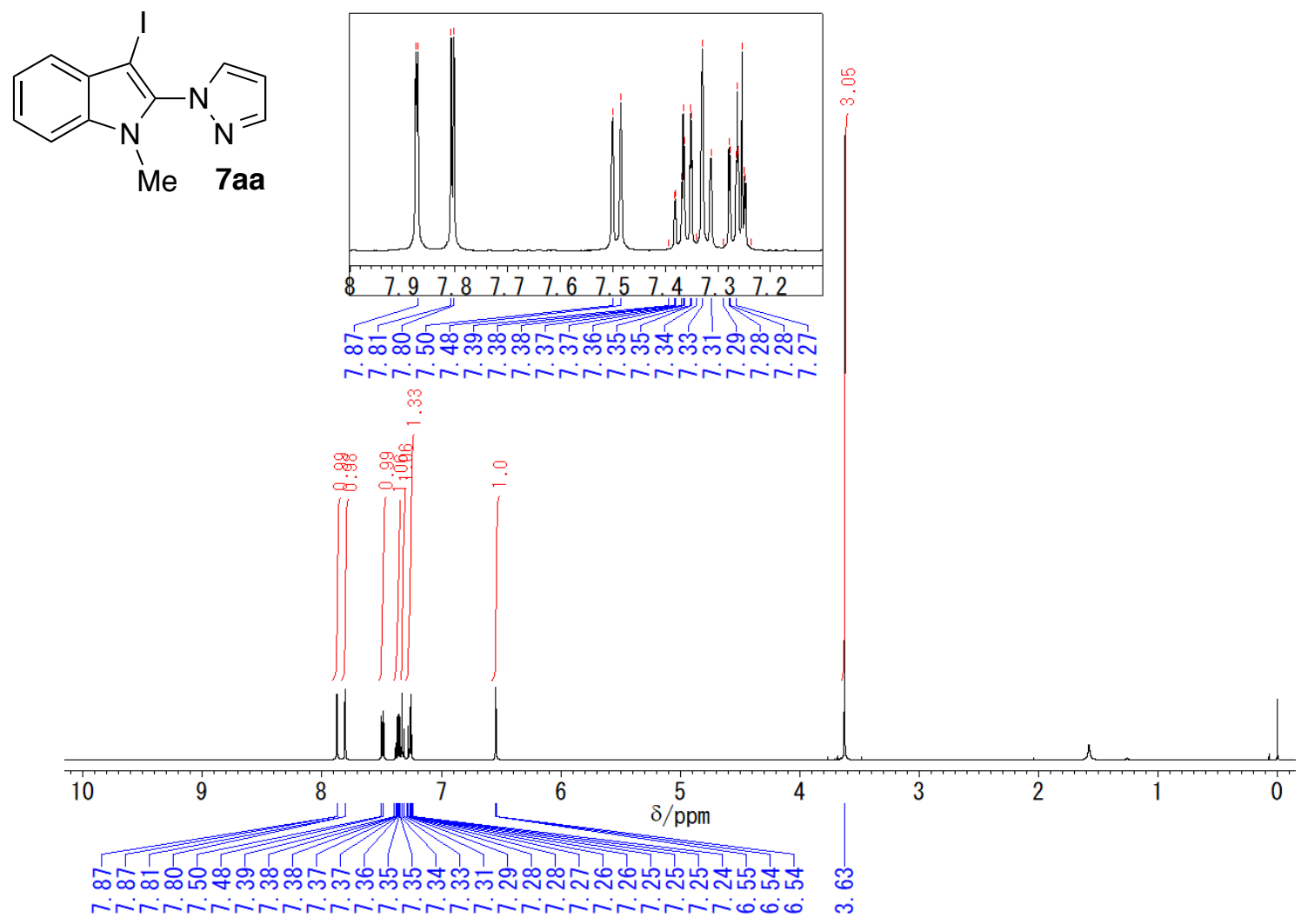

Spectrum S35. ${ }^{1} \mathrm{H}$ NMR $\left(\mathrm{CDCl}_{3}, 500 \mathrm{MHz}\right)$ spectrum of compound 7aa.

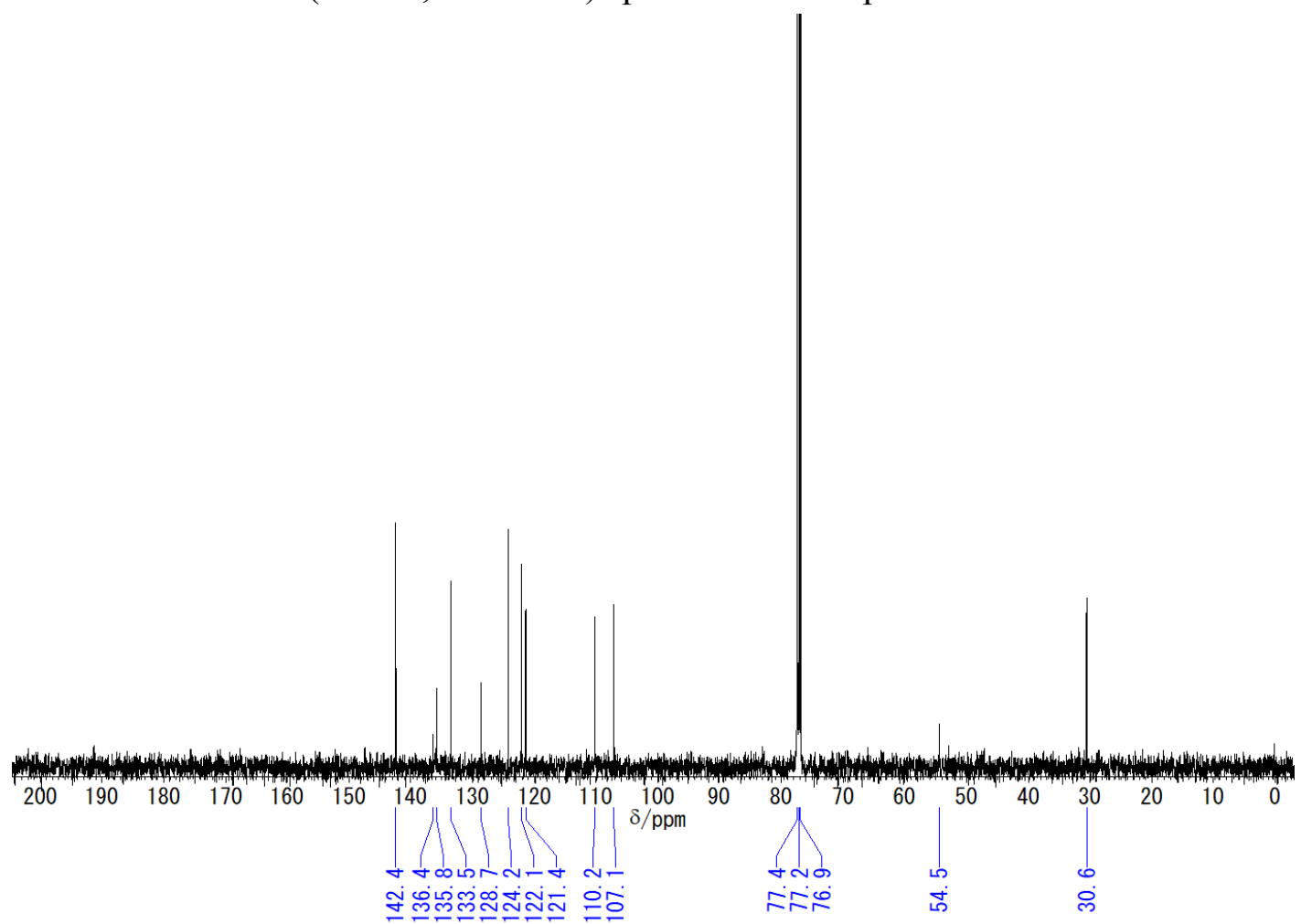

Spectrum S36. ${ }^{13} \mathrm{C}\left\{{ }^{1} \mathrm{H}\right\}$ NMR $\left(\mathrm{CDCl}_{3}, 126 \mathrm{MHz}\right)$ spectrum of compound 7aa. 
<smiles>COc1cc(Sc2c(-n3cncn3)[nH]c3ccccc23)cc(OC)c1OC</smiles>
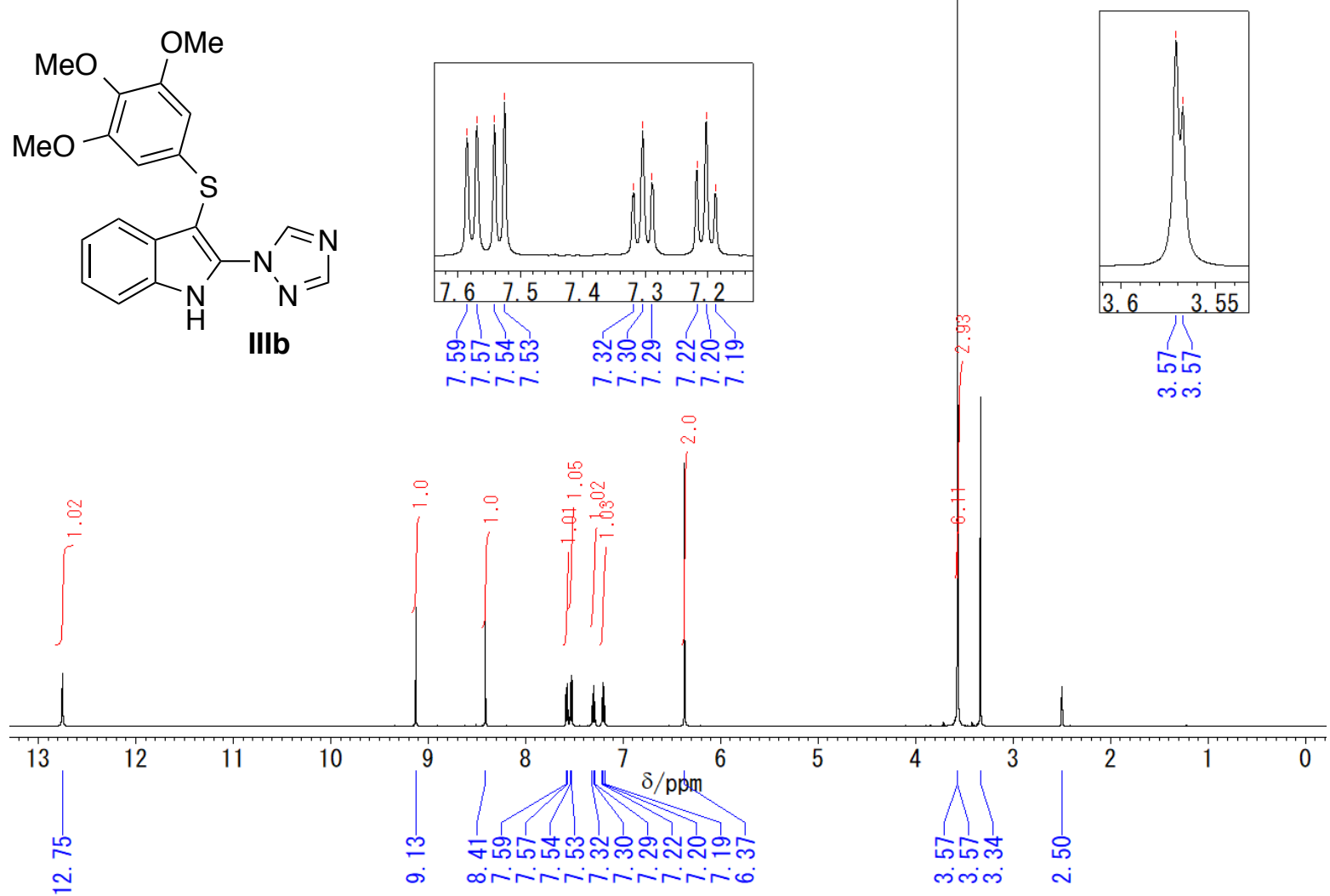

Spectrum S37. ${ }^{1} \mathrm{H}$ NMR (DMSO- $d_{6}, 500 \mathrm{MHz}$ ) spectrum of compound IIIb.

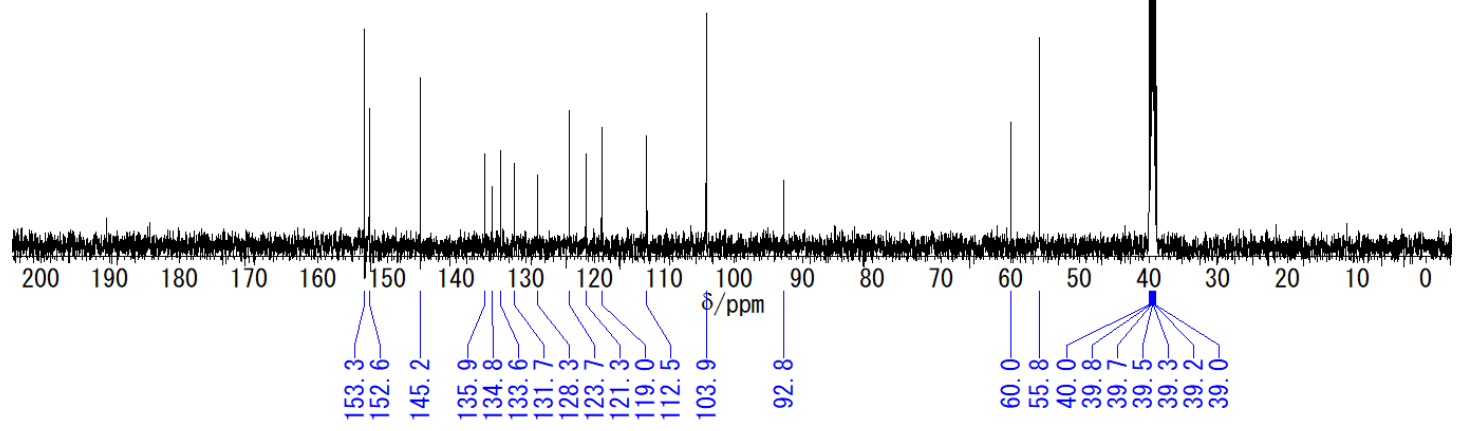

Spectrum S38. ${ }^{13} \mathrm{C}\left\{{ }^{1} \mathrm{H}\right\}$ NMR (DMSO- $d_{6}, 126 \mathrm{MHz}$ ) spectrum of compound IIIb. 


\section{References}

(S1) Müller, F.; Donald, B. M.; Lemuel, D. W. Methods Enzymol., 1971, 18, 453-458.

(S2) Sakai, T.; Kumoi, T.; Ishikawa, T.; Nitta, T.; Iida, H. Org. Biomol. Chem. 2018, 16, 3999-4007.

(S3) Beuleaw D.; Udomsasporn K.; Yotphan S. J. Org. Chem. 2015, 80, 3447-3454.

(S4) Wu, W.-B.; Huang, J.-M. Org. Lett. 2012, 14, 5832-5835.

(S5) Regina, G. L.; Bai, R.; Rensen, W. M.; Cesare, E. D.; Coluccia, A.; Piscitelli, F.; Famiglini, V.; Reggio, A.; Nalli, M.; Pelliccia, S.; Pozzo, E. D.; Costa, B.; Granata, I.; Porta, A.; Maresca, B.; Soriani, A.; Iannitto, M. L.; Santoni, A.; Li, J.; Cona, M. M.; Chen, F.; Ni, Y.; Brancale, A.; Dondio, G.; Vultaggio, S.; Varasi, M.; Mercurio, C.; Martini, C.; Hamel, E.; Lavia, P.; Novellino, E.; Silvestri, R. J. Med. Chem. 2013, 56, 123-149. 